\title{
Strangeness dynamics and transverse pressure in relativistic nucleus-nucleus collisions
}

\author{
E. L. Bratkovskaya, M. Bleicher, M. Reiter, S. Soff, and H. Stöcker \\ Institut für Theoretische Physik, J. W. Goethe Universität, 60054 Frankfurt, Germany \\ M. van Leeuwen \\ NIKHEF, Amsterdam, Netherlands and Lawrence Berkeley National Laboratory, Berkeley, California 94720, USA \\ S. A. Bass \\ Department of Physics, Duke University, Durham, North Carolina 27708, USA \\ and RIKEN BNL Research Center, Brookhaven National Laboratory, Upton, New York 11973, USA \\ W. Cassing \\ Institut für Theoretische Physik, Universität Giessen, 35392 Giessen, Germany
}

(Received 6 February 2004; published 28 May 2004)

\begin{abstract}
We investigate hadron production as well as transverse hadron spectra from proton-proton, proton-nucleus, and nucleus-nucleus collisions from $2 A \mathrm{GeV}$ to $21.3 A \mathrm{TeV}$ within two independent transport approaches, i.e., hadron-string dynamics (HSD) and ultrarelativistic quantum molecular dynamics (UrQMD) that are based on quark, diquark, string, and hadronic degrees of freedom. The comparison to experimental data on transverse mass spectra from $p p, p A$, and $\mathrm{C}+\mathrm{C}$ (or $\mathrm{Si}+\mathrm{Si}$ ) reactions shows the reliability of the transport models for light systems. For central $\mathrm{Au}+\mathrm{Au}(\mathrm{Pb}+\mathrm{Pb})$ collisions at bombarding energies above $\sim 5 A \mathrm{GeV}$, furthermore, the measured $K^{ \pm}$transverse mass spectra have a larger inverse slope parameter than expected from the default calculations. We investigate various scenarios to explore their potential effects on the $K^{ \pm}$spectra. In particular the initial state Cronin effect is found to play a substantial role at top Super Proton Synchrotron (SPS) and Relativistic Heavy Ion Collider (RHIC) energies. However, the maximum in the $\mathrm{K}^{+} / \pi^{+}$ratio at 20-30 $\mathrm{A} \mathrm{GeV}$ is missed by $40 \%$ and the approximately constant slope of the $K^{ \pm}$spectra at SPS energies is not reproduced either. Our systematic analysis suggests that the additional pressure-as expected from lattice QCD calculations at finite quark chemical potential $\mu_{q}$ and temperature $T$ - should be generated by strong interactions in the early prehadronic/partonic phase of central $\mathrm{Au}+\mathrm{Au}(\mathrm{Pb}+\mathrm{Pb})$ collisions.
\end{abstract}

DOI: 10.1103/PhysRevC.69.054907

PACS number(s): 25.75.Dw, 25.75.Ld, 13.60.Le

\section{INTRODUCTION}

The phase transition from partonic degrees of freedom (quarks and gluons) to interacting hadrons is a central topic of modern high-energy physics [1]. In order to understand the dynamics and relevant scales of this transition, laboratory experiments under controlled conditions are presently performed with ultrarelativistic nucleus-nucleus collisions. Hadronic spectra and relative hadron abundances from these experiments reflect important aspects of the dynamics in the hot and dense zone formed in the early phase of the reaction. Here, different quark flavors $(u, d, s, c, b)$ - as constituents of the final hadrons - provide valuable information on the early high density phase due to the substantially different mass scales for the light $(u, d)$, strange $(s)$, and charm $(c)$ or bottom $(b)$ quarks. Furthermore, as has been proposed earlier by Rafelski and Müller [2], in particular the strangeness degree of freedom might play an important role in distinguishing hadronic and partonic dynamics.

Lattice QCD calculations at vanishing quark chemical potential and finite temperature indicate critical energy densities for the formation of a quark-gluon plasma (QGP) of $\sim 0.7-1 \mathrm{GeV} / \mathrm{fm}^{3}[3]$, which might already be achieved at alternating gradient synchrotron (AGS) energies of $\sim 10 \mathrm{~A} \mathrm{GeV}$ for central $\mathrm{Au}+\mathrm{Au}$ collisions [4-6]. Moreover, the recent lattice QCD calculations at finite quark chemical potential $\mu_{q}$ [7] show a rapid increase of the thermodynamic pressure $P$ with temperature above the critical temperature $T_{c}$ for a phase transition (or crossover) to the QGP. The problem is how to relate these numbers to observables from nucleusnucleus collisions. Estimates based on the Bjorken formula [8] for the energy density achieved in central $\mathrm{Au}+\mathrm{Au}$ collisions suggest that the critical energy density for the formation of a QGP of $\sim 0.7-1 \mathrm{GeV} / \mathrm{fm}^{3}$ is by far exceeded during a few $\mathrm{fm} / \mathrm{c}$ in the initial phase of $\mathrm{Au}+\mathrm{Au}$ collisions at relativistic heavy ion collider (RHIC) energies [1]. Accordingly, a phase transition (or crossover) might already happen at much lower bombarding energies.

Experimentally, relativistic nucleus-nucleus collisions have been studied at beam energies from 0.1 to $2 A \mathrm{GeV}$ at the SchwerIonen Synchrotron (SIS), from 2 to $11.6 \mathrm{~A} \mathrm{GeV}$ at the AGS and from 20 to $160 A \mathrm{GeV}$ at the super proton synchrotron (SPS) $[9,10]$. This program has been extended [1] at RHIC with $\mathrm{Au}+\mathrm{Au}$ collisions at invariant energies $\sqrt{s}$ from $\sim 20$ to $200 \mathrm{GeV}$ (equivalent energies in a fixed target experiment: $0.2-21.3 A \mathrm{TeV}$ ).

Whereas many previous experimental studies have focused on longitudinal rapidity distributions for hadrons, the transverse mass (or momentum) spectra of hadrons are presently in the center of interest. On the one hand, a significant suppression of high transverse momentum hadrons in $\mathrm{Au}$ 
+ Au collisions compared to $p p$ is observed at RHIC energies of $\sqrt{s}=200 \mathrm{GeV}$ [11-14]. This suppression is often attributed to the energy loss of highly energetic particles in a hot colored medium (QGP) $[15,16]$. In fact, the recent observation by the PHENIX [12], STAR [13], and BRAHMS [14] collaborations, that a similar suppression is not observed in $d$ $+\mathrm{Au}$ interactions at midrapidities at the same energy, supports this idea. On the other hand, the measured transverse mass $\left[m_{T}=\left(p_{T}^{2}+m^{2}\right)^{1 / 2}\right]$ spectra of hadrons (heavier than pions)

$$
\frac{1}{m_{T}} \frac{d N}{d m_{T}} \sim \exp \left(-\frac{m_{T}}{T}\right),
$$

at AGS, SPS, and RHIC energies show a substantial hardening in central $\mathrm{Au}+\mathrm{Au}$ collisions relative to $p p$ interactions (cf. $[10,17])$. This hardening of the spectra [or increase of the inverse slope parameter $T$ in Eq. (1)] for low transverse mass or momentum is commonly attributed to a strong collective flow, which is absent in the respective $p p$ or $p A$ collisions.

The authors of [18] have proposed to interpret the approximately constant $K^{ \pm}$slopes above $\sim 30 \mathrm{~A} \mathrm{GeV}$-the "step"- as an indication for a phase transition. This interpretation is also based on a rather sharp maximum in the $\mathrm{K}^{+} / \pi^{+}$ ratio at $\sim 20$ to $30 \mathrm{~A} \mathrm{GeV}$ in central $\mathrm{Pb}+\mathrm{Pb}$ collisions (the "horn" [18]). However, the present body of experimental data and our theoretical understanding does not allow for a stringent conclusion based only on the step and horn phenomena. In this paper we will address some further theoretical issues, but would like to point out that more experimental data, for example on collisions in the energy range of $20 \leqslant \sqrt{s} \leqslant 200 \mathrm{GeV}$ and on weakly interacting hadrons, will be needed to clarify these issues from the experimental side.

We will demonstrate in this work, that neither the pressure needed to generate a large collective flow-to explain the hard slopes of the $K^{ \pm}$spectra with a "plateau" at SPS energies-nor the experimental sharp maximum in the $K^{+} / \pi^{+}$ratio at $\sim 20-30 \mathrm{~A} \mathrm{GeV}$ is produced in the present transport models by the interactions of hadrons in the expansion phase of the hadronic fireball. Some aspects of this work have been published in Refs. $[19,20]$ before; however, we here extend the previous studies to RHIC energies and consider a wide variety of dynamical scenarios. In particular we will explore two distinct effects: (i) initial state Cronin enhancement and (ii) heavy resonance formation on the transverse mass spectra of pions and kaons at all bombarding energies from lower AGS to RHIC energies.

In our studies we use two independent relativistic transport models that employ hadronic and string degrees of freedom, i.e., ultrarelativistic quantum molecular dynamics (UrQMD) [21,22] and hadron-string dynamics (HSD) $[23,24]$. They take into account the formation and multiple rescattering of hadrons, and thus dynamically describe the generation of pressure in the hadronic expansion phase. This involves, also, interactions of leading prehadrons that contain a valence quark (antiquark) from a primary "hard" collision (cf. Refs. $[19,25])$. In the parton language, the two transport models employed do not include gluon degrees of freedom explicitly nor gluon-gluon and gluon-quark (antiquark) inter- actions. We will use these models to test, whether the description of nucleus-nucleus reactions in terms of prehadronic, hadronic, and string degrees of freedom and their interactions is sufficient to explain the experimental data.

Our work is organized as follows. In Sec. II we briefly recall the ingredients of the HSD and UrQMD transport approaches and point out the extensions incorporated in UrQMD (versions 2.0 and 2.1). In Sec. III the calculated excitation functions for pions, $K^{ \pm}$mesons and hyperons for central $\mathrm{Au}+\mathrm{Au}$ (or $\mathrm{Pb}+\mathrm{Pb}$ ) collisions from 2 to $21300 \mathrm{~A} \mathrm{GeV}$ are shown (in comparison to the experimental data) as well as selected particle ratios. Sec. IV is devoted to transverse mass spectra of pions and $K^{ \pm}$mesons from $p p$ to $p A$ and central $A A$ reactions in the same energy range. In Sec. V we will discuss various alternative scenarios within the transport calculations to explore their sensitivity to the hadron transverse mass spectra. Sec. VI is devoted to a study of the "Cronin effect" in central $\mathrm{Au}+\mathrm{Au}$ collisions, which we attribute to a "prehadronic" initial state scattering effect that also shows up in $p A$ reactions. Sec. VII includes a comparison of the thermodynamic Lagrange parameters $T$ and $\mu_{B}$ extracted from the UrQMD transport model in the central overlap regime of $\mathrm{Au}+\mathrm{Au}$ collisions with the experimental systematics on chemical freeze-out configurations in the $T, \mu_{B}$ plane as well as recent lattice QCD results. Sec. VIII closes this study with a summary and discussion of open problems.

\section{TRANSPORT MODELS-HSD AND URQMD}

We use two independent relativistic transport approaches for our study that employ hadronic and string degrees of freedom, i.e., UrQMD [21,22] and HSD [23,24,26], in order to obtain an estimate of systematic uncertainties within the transport models. Both approaches take into account the formation and decay of strings as well as the multiple rescattering of hadrons in the expansion phase. These models can be considered as appropriate descriptions, at least for the final stage of an ultrarelativistic nucleus-nucleus collision, where the strongly interacting hadrons dominate. The open question is, however, if these transport models also incorporate the proper degrees of freedom in the very early phase of heavy ion collisions. We stress that transport calculations allow for a systematic study of the change in the dynamics from elementary baryon-baryon or meson-baryon collisions to proton-nucleus reactions or from peripheral to central nucleus-nucleus collisions in a unique way without change in parameters. This is of central importance since the assumptions of thermal and chemical equilibrium-as used in hydrodynamical or statistical models [27] — do not necessarily hold in all these reactions and the transport studies allow us to explore the amount of (thermal or chemical) equilibrium reached [28-30].

The default UrQMD 1.3 transport approach $[21,22]$ includes all baryonic resonances up to masses of $2 \mathrm{GeV}$ as well as mesonic resonances up to $1.9 \mathrm{GeV}$ as tabulated by the particle data group [31]. For hadronic continuum excitations a string model is used with meson formation times of the order of $1-2 \mathrm{fm} / c$ depending on the momentum and 
energy of the created hadron. For the present study — and the particular aim to address also jet production and fragmentation at RHIC energies - we additionally have incorporated PYTHIA (v 6.1) [32] in UrQMD 1.3 similar to HSD. This extended version we denote as UrQMD 2.0.

Moreover, to explore the physical consequences of higher mass states as implemented in the relativistic-quantummolecular-dynamics model (RQMD) [33], we have introduced in UrQMD additional high mass resonances that are explicitly produced and propagated in $s$-channel processes with invariant masses up to $\sqrt{s}<3 \mathrm{GeV}$. We do not assume these states to be excited in baryon-baryon collisions. In practical terms: If the mass of the produced mesonic or baryonic resonance is above $\sim 2 \mathrm{GeV}$, i.e, in the continuum, the formed resonance state is not fragmented as a string object like in UrQMD 1.3 or UrQMD 2.0. We recall that a string of invariant mass $2 \leqslant M \leqslant 3 \mathrm{GeV}$ essentially decays to a few pions, a vector meson +pions, etc. Instead, this high mass state is treated as a quasiparticle that decays according to the branching ratios of the resonances with a mass of $1.9-2.0 \mathrm{GeV}$ (as implemented in UrQMD). We recall that baryon resonances $\sim 1.9 \mathrm{GeV}$ dominantly decay to the $\pi$ $+N$ channel and, with some lower probability, to the $\rho(\omega)$ $+N$ final state. In this way light meson emission is suppressed by about $25 \%$ compared to a string of the same invariant mass. We note that this recipe is still in line with the available $\pi p \rightarrow \pi X$ data on pion multiplicities. Furthermore, since the high mass resonances decay isotropically in their rest frame, this leads to an enhancement of transverse flow (cf. Sec. V E). Furthermore, we use isotropic angular distributions for elastic meson-baryon interactions below $\sqrt{s_{\mathrm{mb}}}=6 \mathrm{GeV}$. In this work we will denote this scenario as UrQMD 2.1.

In the HSD approach nucleons, $\Delta$ 's, $N^{*}(1440), N^{*}(1535)$, $\Lambda, \Sigma$ and $\Sigma^{*}$ hyperons, $\Xi$ 's, $\Xi^{*}$ 's and $\Omega$ 's as well as their antiparticles are included on the baryonic side whereas the $0^{-}$ and $1^{-}$octet states are included in the mesonic sector. Inelastic hadron-hadron collisions with energies above $\sqrt{s}$ $\simeq 2.6 \mathrm{GeV}$ are described by the FRITIOF model [34] (incorporating PYTHIA v 5.5 with JETSET v 7.3 for the production and fragmentation of jets [32]), whereas low energy hadron-hadron collisions are modeled in line with experimental cross sections [23,24,35].

Both transport approaches reproduce the nucleon-nucleon, meson-nucleon, and meson-meson cross section data in a wide kinematic range $[19,21,22]$. Therefore, we expect that the "hadronic" scattering mechanisms are sufficiently under control. However, in nucleus-nucleus reactions, further elastic and inelastic processes on the parton level might occur that-beyond the interactions of "leading" quarks/diquarks-are not included in the studies below. We mention that (pQCD-like) partonic scattering is incorporated, e.g., in the multiphase transport model (AMPT) [30], which is currently employed from upper SPS to RHIC energies, but is not suitable for lower energies.

\section{EXCITATION FUNCTIONS}

Whereas the underlying concepts of the transport theoretical models are very similar, the actual realizations differ

\footnotetext{
${ }^{1}$ The explicit value depends on the type of resonance.
}

considerably. A systematic analysis of results from both models and experimental data for central nucleus-nucleus collisions from 2 to $160 \mathrm{~A} \mathrm{GeV}$ has shown that the "longitudinal" rapidity distributions of protons, pions, kaons, antikaons, and hyperons are quite similar in both models and in reasonable agreement with available data [19]. We here extend the latter studies to RHIC energies, but focus on total and midrapidity hadron yields as well as particle ratios. For completeness we include the results from Ref. [19] in Figs. $1-3$.

\section{A. Pion and strange particle yields}

Figure 1 shows the excitation function of $\pi^{+}, \pi^{-}, K^{+}, K^{-}$, and $\Lambda+\Sigma^{0}$ yields from $5 \%$ central (AGS energies, SPS at $160 \mathrm{~A} \mathrm{GeV}$ and at RHIC energies), $7 \%$ central (20, 30, 40, and $80 \mathrm{~A} \mathrm{GeV}$ ), $10 \%$ central for $\Lambda+\Sigma^{0}$ at $160 A \mathrm{GeV} \mathrm{Au}$ $+\mathrm{Au}$ (AGS and RHIC) or $\mathrm{Pb}+\mathrm{Pb}$ (SPS) collisions in comparison to the experimental data from Refs. [36-38] (AGS), $[9,39,41]$ (SPS), and [42-44] (RHIC) for midrapidity (left column) and rapidity integrated yields (right column). The solid lines with open squares show the results from HSD, the dashed lines with open triangles indicate the calculations with UrQMD 1.3, and the long dashed lines with crosses depict the UrQMD 2.1 results.

We note in passing, that the corrections to the pion and kaon yields due to the weak decay of resonances have not been included in our calculations in line with the experimental analysis of the NA49 Collaboration (cf. Refs. [9,10]). However, the experimental subtraction of weak decays is problematic and might have an impact on the measured kaon to pion ratio as pointed out in Ref. [45]. This uncertainty has to be kept in mind throughout the following analysis.

As already demonstrated in Ref. [19] the agreement between the transport models HSD (solid lines) and UrQMD 1.3 (dashed lines) is rather good, as well as with the data available. The exception are the pion yields at the highest AGS energy and lower SPS energies, which are overestimated by both approaches (cf. [19]). This is demonstrated in more detail in Fig. 2, where the $\pi^{ \pm}$and $K^{ \pm}$yields (as in Fig. 1) are displayed in the energy range up to $80 \mathrm{~A} \mathrm{GeV}$ on a linear scale. The overestimation of the pion yields (in $4 \pi$ ) in this energy range is slightly more pronounced for UrQMD 1.3 than for HSD especially at the higher SPS energy. On the other hand, both transport models fall slightly low in the $K^{+}$ yields at 30 and $40 \mathrm{~A} \mathrm{GeV}$. The results from version UrQMD 2.1 (long dashed lines) differ from UrQMD 1.3 essentially in the following aspect: The pion multiplicity at AGS and SPS energies is reduced by about 25\% (cf. Sec. II) due to the specific decay scheme of higher mass resonances (and is now in better agreement with the data). On the other hand, UrQMD 2.1 gives a lower amount of kaons and especially antikaons (in $4 \pi$ ) than the "default" HSD and UrQMD 1.3 approaches.

At RHIC energies the UrQMD 1.3 calculations show a $\sim 30 \%$ higher pion yield at midrapidity than HSD while the differences between the $4 \pi$ integrated yields are of the order of $10 \%$, both between the models and compared to the data. The implementation of higher mass states in UrQMD 2.1 (cf. 


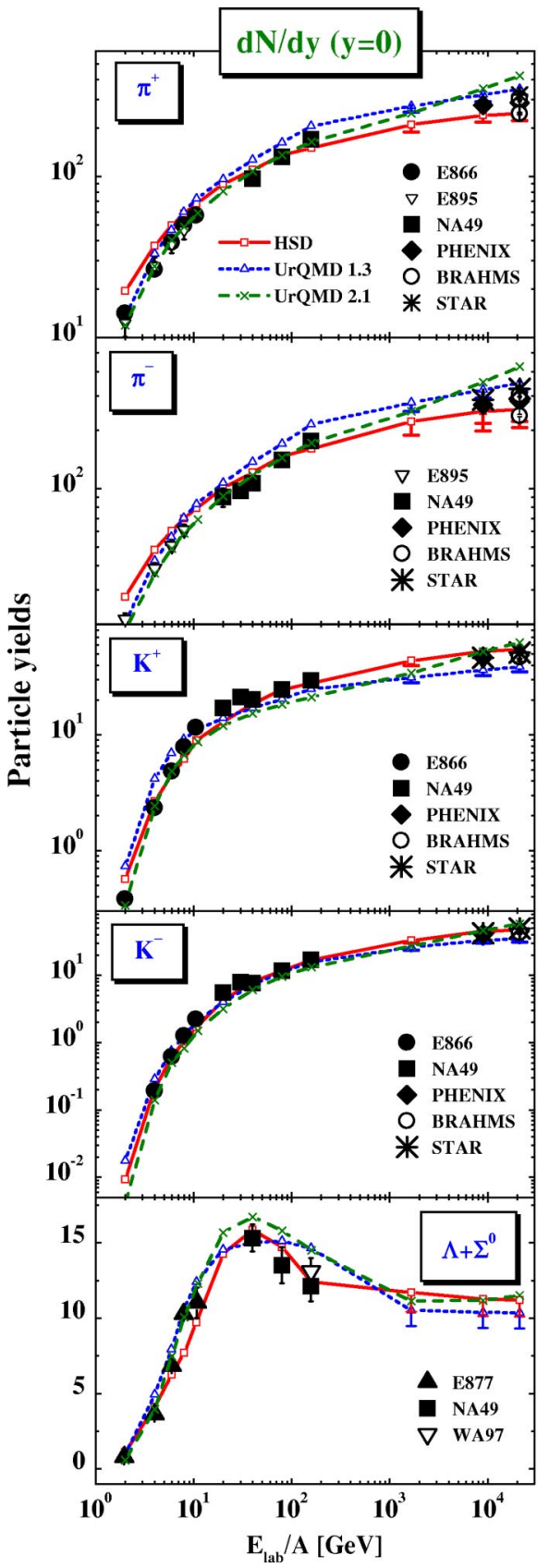

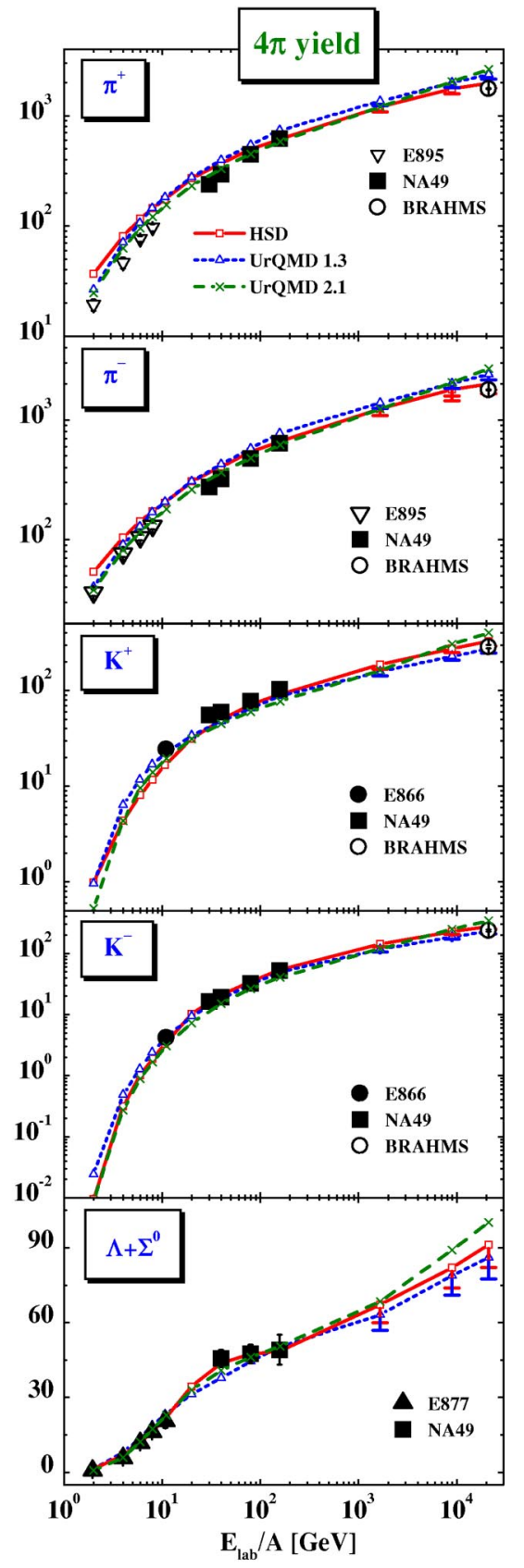

FIG. 1. (Color online) The excitation function of $\pi^{+}, \pi^{-}, K^{+}$, $K^{-}$, and $\Lambda+\Sigma^{0}$ yields from $5 \%$ central (AGS energies, SPS at $160 \mathrm{~A} \mathrm{GeV}$ and at RHIC energies), $7 \%$ central $(20,30,40$, and $80 \mathrm{~A} \mathrm{GeV}), 10 \%$ central for $\Lambda$ $+\Sigma^{0}$ at $160 \mathrm{~A} \mathrm{GeV} \mathrm{Au+Au} \mathrm{(AGS}$ and $\mathrm{RHIC}$ ), or $\mathrm{Pb}+\mathrm{Pb}$ (SPS) collisions in comparison to the experimental data from Refs. [36-38] (AGS), [9,39-41] (SPS), and [42-44] (RHIC) for midrapidity (left column) and rapidity integrated yields (right column). The solid lines with open squares show the results from HSD whereas the dashed lines with open triangles and the long dashed lines with crosses indicate the results from UrQMD 1.3 and UrQMD 2.1, respectively. The lower theoretical errorbars at RHIC energies correspond to the yields for $10 \%$ central events.
Sec. II)-together with the jet production described by PYTHIA-leads to an overestimation of pions, kaons, and hyperons at RHIC energies.

Note that the maximum in the $\Lambda+\Sigma^{0}$ yield at midrapidity (lower left part of Fig. 1) for $\sim 30 \mathrm{~A} \mathrm{GeV}$ is reproduced by both transport approaches. However, this maximum disappears in the $4 \pi$ integrated yields in line with the data (cf. lower right part of Fig. 1). Within the transport models the maximum in the $\Lambda+\Sigma^{0}$ yield at midrapidity can be attributed to a large extent to a change from baryon-dominated dynamics at AGS energies to meson-dominated dynamics at the higher SPS energies.

\section{B. $K^{+} / \pi^{+}, K^{-} / \pi^{-}$, and $\left(\Lambda+\Sigma^{0}\right) / \pi$ ratios}

In Fig. 3 we present the excitation function of the particle ratios $K^{+} / \pi^{+}, K^{-} / \pi^{-}$, and $\left(\Lambda+\Sigma^{0}\right) / \pi$ from $5 \%$ central (AGS energies, SPS at $160 \mathrm{~A} \mathrm{GeV}$ and at RHIC energies), $7 \%$ central $(20,30,40$, and $80 \mathrm{~A} \mathrm{GeV}), 10 \%$ central for $\Lambda+\Sigma^{0}$ at $160 A \mathrm{GeV} \mathrm{Au}+\mathrm{Au}$ (AGS and RHIC) or Pb+ Pb (SPS) collisions in comparison to the experimental data from Refs. [36-38] (AGS), [9,39-41] (SPS), and [42-44] (RHIC) for midrapidity yields (left column) and rapidity integrated yields (right column). The solid lines with open squares show the results from HSD, the dashed lines with open triangles indicate the UrQMD 1.3 calculations, whereas the long dashed lines with crosses correspond to the UrQMD 2.1 results. The deviations between the transport models and the data are most pronounced for the midrapidity ratios (left column) since the ratios are very sensitive to actual rapidity spectra. The $K^{+} / \pi^{+}$ratio in UrQMD 1.3 shows a maximum at $\sim 8.4 \mathrm{~A} \mathrm{GeV}$ and then drops to a roughly constant ratio of 0.12 at top SPS and RHIC energies. UrQMD 2.1 follows the 


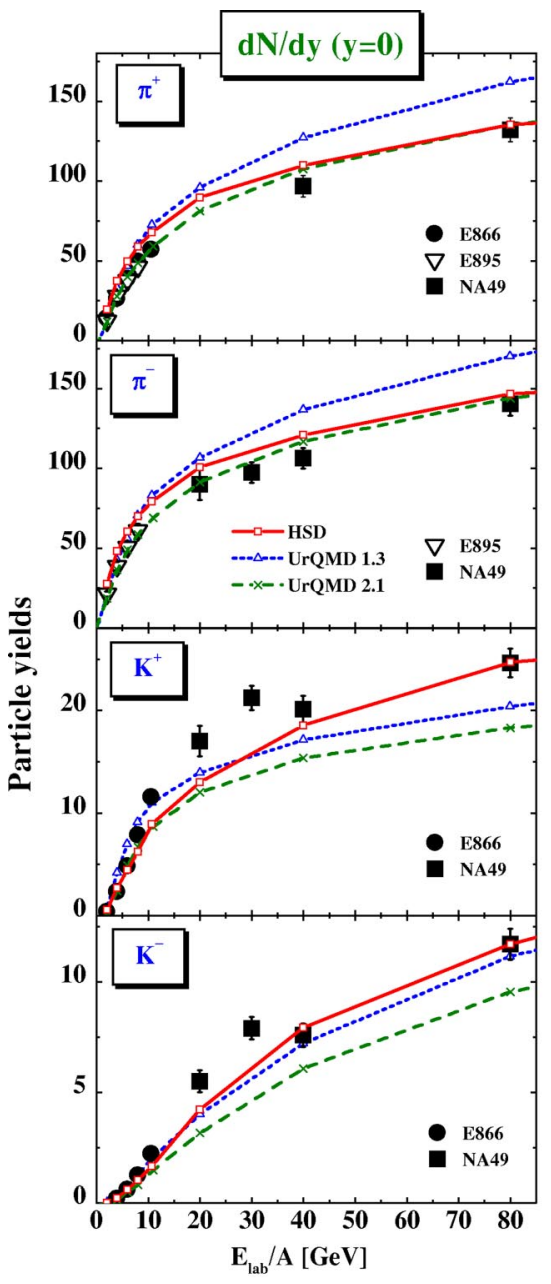

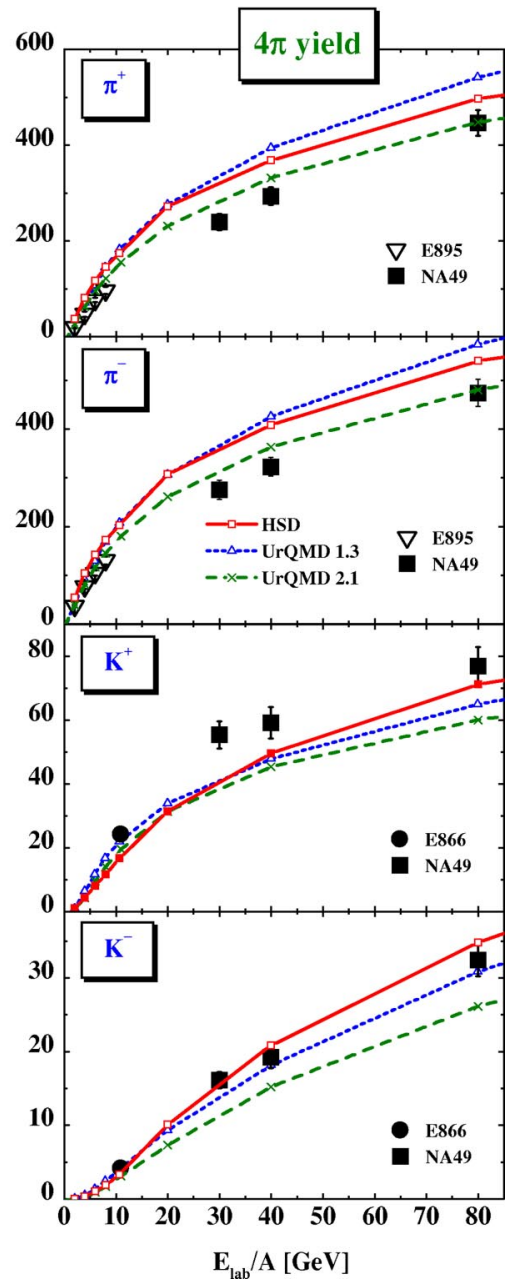

FIG. 2. (Color online) The excitation function of $\pi^{+}, \pi^{-}, K^{+}$, and $K^{-}$mesons (as in Fig. 1), but up to $80 \mathrm{~A} \mathrm{GeV}$ on a linear scale. results from UrQMD 1.3 at AGS and SPS energies, but then increases slightly up to RHIC energies. In case of HSD, a continuously rising ratio with bombarding energy is found for the midrapidity ratios. This rise up to the full RHIC energy is essentially due to an underestimation of the pion yield at midrapidity (cf. Fig. 1 in Ref. [48]). The $4 \pi$ ratio in HSD, which is approximately constant from top SPS to RHIC energies, is larger than the ratio from UrQMD 1.3 due to the lower amount of pion production and a slightly higher $K^{+}$ yield (cf. Fig. 1). We note that the lower amount of pions in HSD is essentially due to an energy-density cut (in the local rest frame), which does not allow hadron formation when the energy density is above $1 \mathrm{GeV} / \mathrm{fm}^{3}$.

Despite this seemingly good description of the separate particle yields, the experimentally observed maximum in the $\mathrm{K}^{+} / \pi^{+}$ratio at 20-30 $\mathrm{A} \mathrm{GeV}$ is not reproduced in the models. Due to strangeness conservation, the same arguments hold for the $\left(\Lambda+\Sigma^{0}\right) / \pi$ ratio, where the pronounced experimental maxima are underestimated due to the excess of pions in the transport models at top AGS energies (for HSD) and above $\sim 5 A \mathrm{GeV}$ (for UrQMD 1.3). Here UrQMD 2.1 performs better in the ratios due to the lower amount of pions produced by the high resonance decays. Since the $K^{-}$yields are well described by all approaches (cf. Fig. 1) the deviations in the $K^{-} / \pi^{-}$ratios at SPS and RHIC energies in UrQMD 1.3 indeed can be traced back to the excess of pions.
This suggests that the entropy production, which is reflected in the pion to baryon ratio at these energies, is overestimated in UrQMD 1.3 (and HSD) above $\sim 5 A \mathrm{GeV}$ in central $\mathrm{Au}$ + Au collisions. Note that in UrQMD 2.1 the entropy production is reduced by the generation of high mass states in $s$ channels, which essentially decay by emitting a lower amount of pions.

It has been discussed in the community [49], that the maximum in the $K^{+} / \pi^{+}$ratio might be due to isospin effects in $\mathrm{Au}+\mathrm{Au}(\mathrm{Pb}+\mathrm{Pb})$ collisions arising from a different rapidity dependence of $u$ and $d$ quarks in $p p, p n$, and $n n$ collisions. We here address this issue and show in Fig. 4 the influence of isospin effects on the kaon to pion ratio. The left part of Fig. 4 displays the excitation function of the $\left\langle\pi^{-}\right\rangle /\left\langle\pi^{+}\right\rangle$ratio from central $\mathrm{Au}+\mathrm{Au}(\mathrm{Pb}+\mathrm{Pb})$ collisions calculated within HSD (solid line with open squares) and UrQMD 1.3 (solid line with open triangles). The right-hand panel shows both the isospin-sensitive $\left\langle K^{+}\right\rangle /\left\langle\pi^{+}\right\rangle$ratio (open symbols) and the (approximately) isospin-insensitive $2\left\langle K^{+}\right\rangle /\left(\left\langle\pi^{+}\right\rangle+\left\langle\pi^{-}\right\rangle\right)$ratio (solid symbols). The HSD results are shown as squares connected by solid lines while the UrQMD 1.3 results are represented as dashed lines with triangles. The larger solid symbols indicate the experimental results for $\left\langle K^{+}\right\rangle /\left\langle\pi^{+}\right\rangle$from Refs. [9,36,41,42] and the large open triangles correspond to the ratio $2\left\langle K^{+}\right\rangle /\left(\left\langle\pi^{+}\right\rangle+\left\langle\pi^{-}\right\rangle\right)$ from Ref. [47]. As seen from Fig. 4, the calculated (as well 

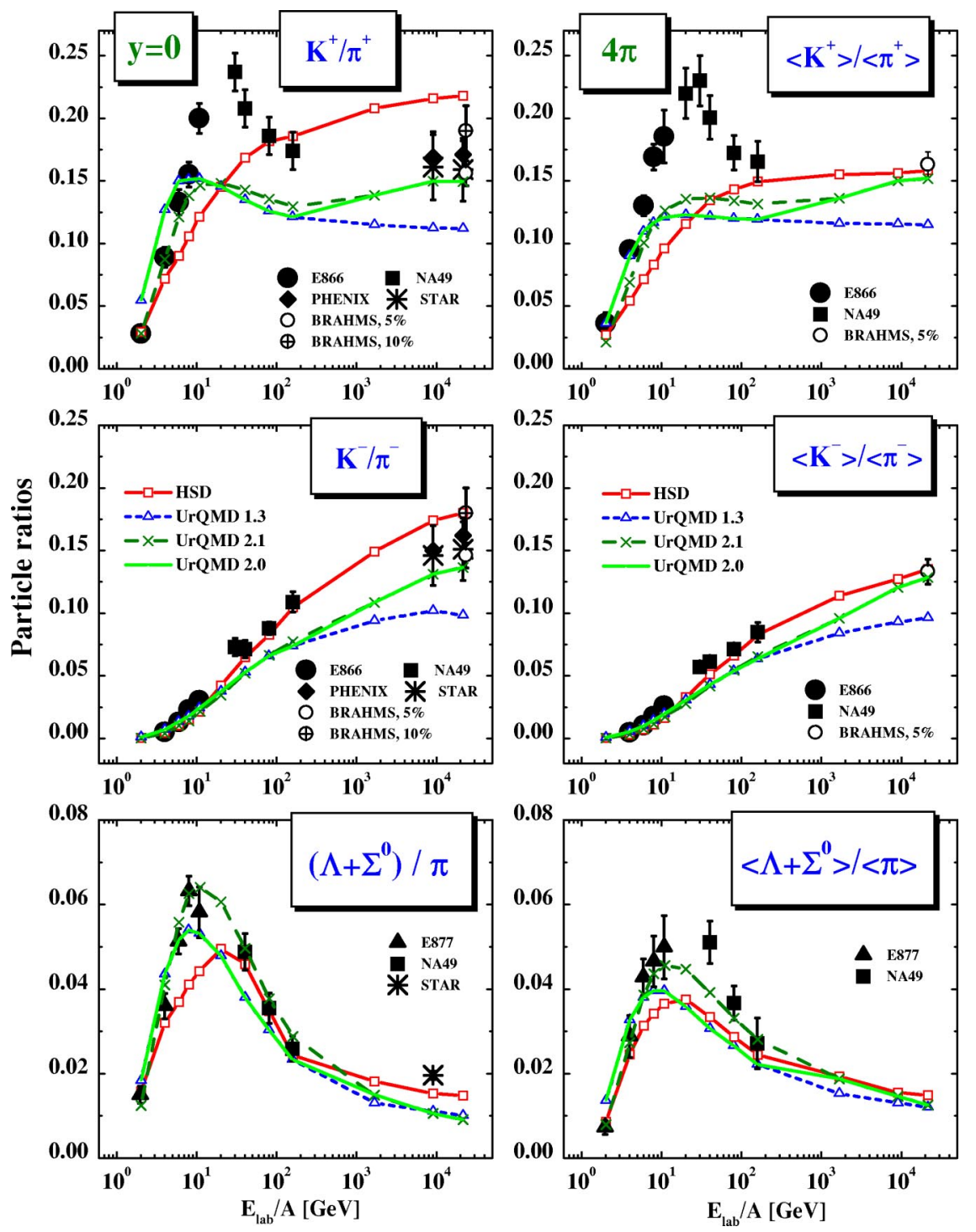

FIG. 3. (Color online) The excitation function of $K^{+} / \pi^{+}, K^{-} / \pi^{-}$, and $\left(\Lambda+\Sigma^{0}\right) / \pi$ ratios from $5 \%$ central (AGS energies, SPS at $160 \mathrm{~A} \mathrm{GeV}$ and at RHIC energies), $7 \%$ central $(20,30,40$, and $80 \mathrm{~A} \mathrm{GeV}), 10 \%$ central for $\Lambda$ $+\Sigma^{0}$ at $160 A \mathrm{GeV} \mathrm{Au}+\mathrm{Au}$ (AGS and $\mathrm{RHIC}$ ), or $\mathrm{Pb}+\mathrm{Pb}$ (SPS) collisions in comparison to the experimental data from Refs. [36,38] (AGS), [9,39-41] (SPS), and [42-44] (RHIC) for midrapidity (left column) and rapidity integrated yields (right column). The solid lines with open squares show the results from HSD whereas the dashed lines with open triangles and the long dashed lines with crosses indicate the results from UrQMD 1.3 and UrQMD 2.1, respectively.
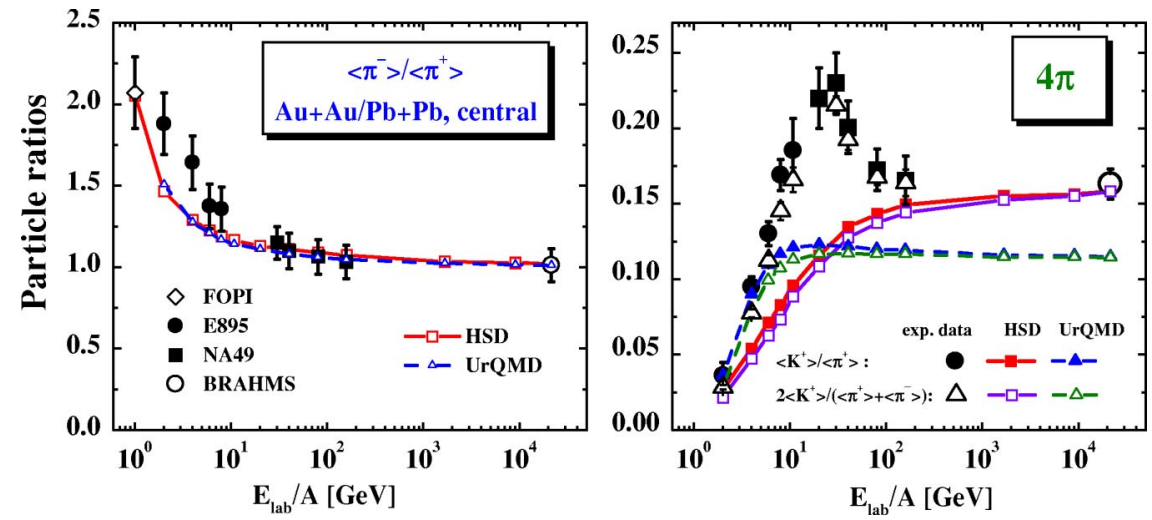

FIG. 4. (Color online) The excitation function of the $\left\langle\pi^{-}\right\rangle /\left\langle\pi^{+}\right\rangle$(left-hand side) and $\left\langle K^{+}\right\rangle /\langle\pi\rangle$ ratios (right-hand side) from central Au $+\mathrm{Au}($ or $\mathrm{Pb}+\mathrm{Pb}$ ) collisions. Left part: the HSD results are shown by the solid line with open squares, whereas the UrQMD 1.3 calculations are displayed in terms of the solid line with open triangles. The experimental data have been taken from Refs. [9,37,42,46]. Right part: the solid line with solid squares (triangles) (cf . Fig. 3) corresponds to the HSD (UrQMD 1.3) calculations for $\left\langle K^{+}\right\rangle /\left\langle\pi^{+}\right\rangle$(as well as the experimental data from Refs. [9,36,41,42], while the solid with open squares (triangles) shows the calculated $2\left\langle K^{+}\right\rangle /\left(\left\langle\pi^{+}\right\rangle+\left\langle\pi^{-}\right\rangle\right)$ratio in comparison to the experimental data (open triangles) from Ref. [47]. 

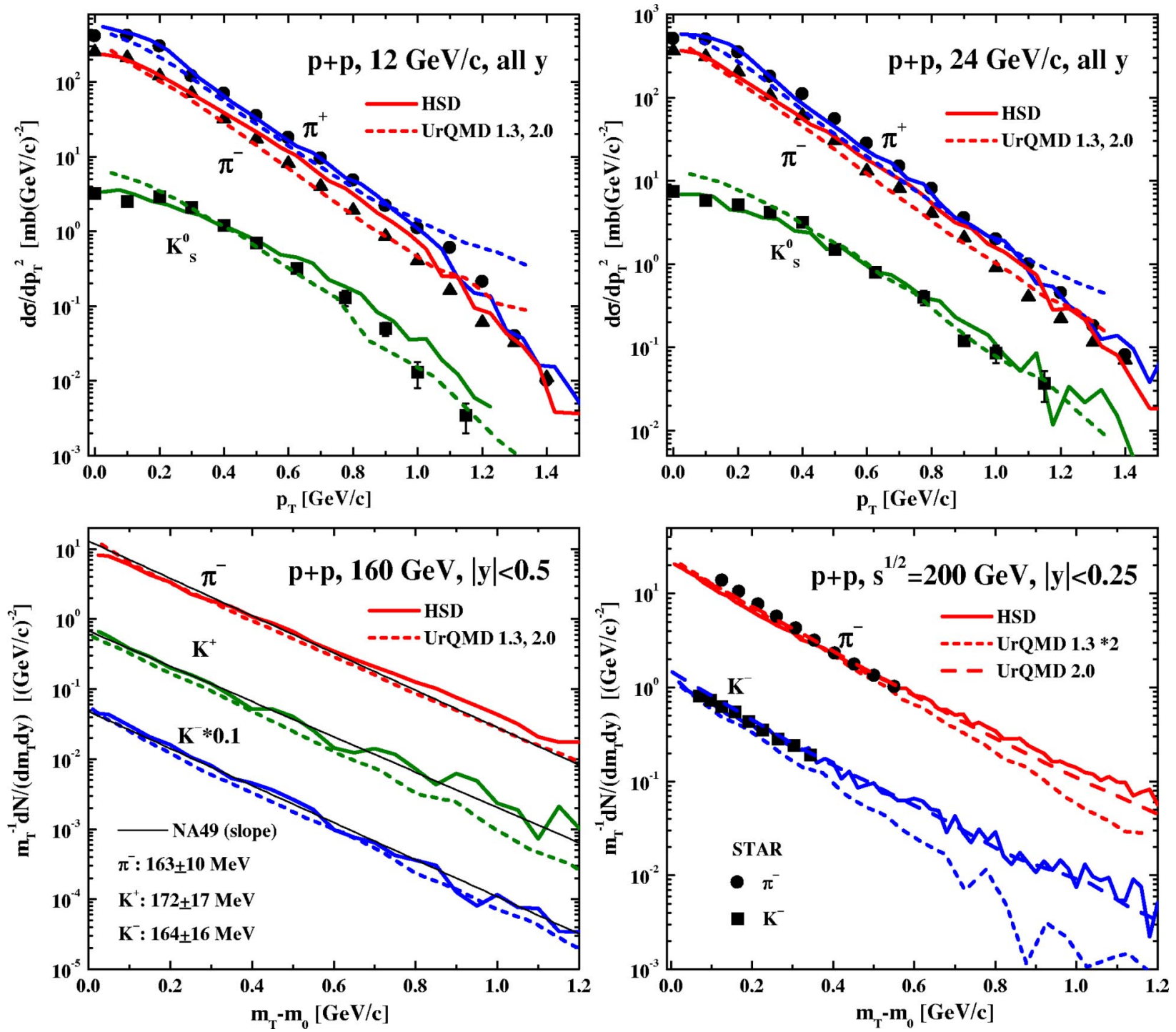

FIG. 5. (Color online) Upper part: comparison of the differential cross sections for $\pi^{+}, \pi^{-}$, and $K_{S}^{0}$ from $p p$ reactions at 12 and $24 \mathrm{GeV} / c$ from HSD (solid lines) and UrQMD 1.3 (=UrQMD 2.0) (dashed lines) with the data from Ref. [50]. Lower part (left): the transverse mass spectra at midrapidity for $\pi^{-}, K^{+}$, and $K^{-}(\times 0.1)$ from $p p$ reactions at a bombarding energy $160 \mathrm{GeV}$ from HSD (solid lines) and UrQMD 1.3 (dashed lines). The thin lines correspond to fits of the experimental slope parameters $163 \pm 10 \mathrm{MeV}$ for $\pi^{-}, 172 \pm 17 \mathrm{MeV}$ for $K^{+}$, and $164 \pm 16 \mathrm{MeV}$ for $K^{-}$from Ref. [68]. Lower part (right): the transverse mass spectra at midrapidity for $\pi^{-}$and $K^{-}$from $p p$ reactions at $\sqrt{s}=200 \mathrm{GeV}$ from HSD (solid lines) and UrQMD 1.3 (dashed lines, multiplied by a factor 2) as well as UrQMD 2.0 (=UrQMD 2.1) (long dashed lines). The full symbols indicate the data from the STAR Collaboration [44].

as experimentally measured) isospin-insensitive ratio $2\left\langle K^{+}\right\rangle /\left(\left\langle\pi^{+}\right\rangle+\left\langle\pi^{-}\right\rangle\right)$is only slightly lower than the isospinsensitive $\left\langle K^{+}\right\rangle /\left\langle\pi^{+}\right\rangle$ratio. This result is due to the approximately constant $\left\langle\pi^{-}\right\rangle /\left\langle\pi^{+}\right\rangle$ratio above $\sim 6 A \mathrm{GeV}$ [left-hand side (1.h.s.) of Fig. 4] in the transport calculations as well as in the data. We thus conclude that isospin effects cannot be responsible for the maximum in the $\left\langle K^{+}\right\rangle /\langle\pi\rangle^{+}$ratio [righthand side (r.h.s.) of Fig. 4].

\section{TRANSVERSE MASS SPECTRA}

Despite the relatively good agreement of data and transport calculations in the longitudinal direction [19], significant deviations are found in the transverse direction as pointed out in Ref. [20] before. This demonstrates that longitudinal rapidity distributions are less sensitive to the hadronic interaction channels employed in the transport models. We here present a more complete and systematic analysis up to the full RHIC energy of $\sqrt{s}=200 \mathrm{GeV}$ and investigate alternative scenarios. We start with benchmark tests for the transport calculations for $p p$ and $p A$ reactions.

\section{A. $p p$ and $p A$ reactions}

Our results for the transverse mass (or momentum) spectra of pions and kaons from $p p$ reactions are presented in Fig. 5. The upper part includes the comparison of the differential cross sections for $\pi^{+}, \pi^{-}$, and $K_{S}^{0}$ from $p p$ reactions at 
12 and $24 \mathrm{GeV} / c$ from HSD (solid lines) and UrQMD 1.3 (dashed lines) with the data from Ref. [50]. The lower left part of Fig. 5 shows the transverse mass spectra at midrapidity for $\pi^{-}, K^{+}$, and $K^{-}(\times 0.1)$ from $p p$ reactions at a bombarding energy of $160 \mathrm{GeV}$ from HSD (solid lines) and UrQMD 1.3 (dashed lines). The thin lines here correspond to fits with the experimental slope parameters $163 \pm 10 \mathrm{MeV}$ for $\pi^{-}, 172 \pm 17 \mathrm{MeV}$ for $K^{+}$, and $164 \pm 16 \mathrm{MeV}$ for $K^{-}$from Ref. [68]. The lower right part of Fig. 5 displays the transverse mass spectra at midrapidity for $\pi^{-}$and $K^{-}$from $p p$ reactions at $\sqrt{s}=200 \mathrm{GeV}$ from HSD (solid lines), UrQMD 1.3 (dashed lines, multiplied by a factor 2), and UrQMD 2.0 (2.1) (long dashed lines). The full symbols, furthermore, stand for the data from the STAR Collaboration [44]. The description of the transverse mass spectra for pions and kaons is reasonably good in both approaches up to $\sqrt{s}$ $=200 \mathrm{GeV}$. We note in passing that the inclusion of PYTHIA (v 6.1) in UrQMD 2.0 and UrQMD 2.1 is essential in order to achieve a good description of the data since the contribution from jet fragmentation is essential at RHIC energies. Without jet production and fragmentation the UrQMD model (version 1.3) underestimates the high momentum spectra severely (similar to the relativistic quantum molecular dynamics (RQMD) model [33,51]).

We continue with $p A$ reactions and show in the left part of Fig. 6 the results for the inverse slope parameters $T$ in (1) for $\pi^{ \pm}, K^{+}$, and $K^{-}$mesons at midrapidity from $p A$ reactions at $14.6 \mathrm{GeV} / c(A=\mathrm{Be}, \mathrm{Al}, \mathrm{Cu}, \mathrm{Au})$, whereas the right part includes the results at $450 \mathrm{GeV} / c(A=\mathrm{Be}, \mathrm{S}, \mathrm{Pb})$. The inverse slope parameters from HSD are shown as open symbols connected by solid lines and from UrQMD 1.3 (2.0) by open symbols (with crosses) connected by dashed lines. The full symbols (1.h.s.) correspond to the midrapidity data $\left(\left\langle y_{l a b}\right\rangle\right.$ $=1.5,1.7,1.9)$ from the E802 Collaboration [52] while the NA44 data [53] at $2.4 \leqslant y_{\mathrm{lab}} \leqslant 3.5, p_{T} \leqslant 0.84 \mathrm{GeV} / c$ for $K^{+}, K^{-}$and at $2.4 \leqslant y_{\mathrm{lab}} \leqslant 3.0, p_{T}=0.3 / 1.2 \mathrm{GeV} / c$ (full diamonds) and $3.1 \leqslant y_{\mathrm{lab}} \leqslant 4.0, p_{T} \leqslant 0.64 \mathrm{GeV} / c$ (full squares) for $\pi^{+}$are given on the right-hand side.

The overall reproduction of the transverse slope parameters at AGS and SPS energies for targets from $\mathrm{Be}$ to $\mathrm{Pb}$ by both transport models is sufficiently accurate. The inverse slope parameters from UrQMD 1.3 and HSD differ for pions and antikaons by about $10 \%$ at AGS energies. These differences can be traced back to the different production channels in the transport models at lower energies: in HSD the mesons are essentially produced by string decays, which are strongly forward peaked in momentum, whereas in UrQMD 1.3 they partly stem from high mass resonance decays with an isotropic decay in momentum space. At SPS energies the meson production in both models proceeds via string formation and decay and leads to very similar results. This also holds for RHIC energies where, additionally, jet production and fragmentation contributes with increasing bombarding energy.

We note in passing that when including the initial state "Cronin effect" (cf. Sec. VI), the inverse slope parameters $T$ in Fig. 6 increase by less than 5\% even for the $\mathrm{Pb}$ target at $450 \mathrm{GeV} / c$. At $14.6 \mathrm{GeV} / c$ there is no effect (within statistics) for all targets from $\mathrm{Be}$ to $\mathrm{Au}$.

\section{B. A + A reactions}

We continue with nucleus-nucleus collisions and recall that the experimental $m_{T}$ spectra (at midrapidity) from central $\mathrm{C}+\mathrm{C}$ and $\mathrm{Si}+\mathrm{Si}$ collisions at $160 \mathrm{~A} \mathrm{GeV} \mathrm{[68]} \mathrm{are} \mathrm{well}$ described by HSD and UrQMD 1.3. This holds for the $\pi^{-}$, $K^{+}$, as well as $K^{-}$spectra (cf. Fig. 1 in Ref. [20]).

This situation changes for central collisions of heavy nuclei like $\mathrm{Au}+\mathrm{Au}$ or $\mathrm{Pb}+\mathrm{Pb}$. In Fig. 7 we display the calculated transverse mass spectra at midrapidity from HSD (solid lines) and UrQMD 2.0 (dashed lines) for $\pi^{+}, K^{+}$, and $K^{-}(\times 0.1)$ from central $\mathrm{Au}+\mathrm{Au}(\mathrm{Pb}+\mathrm{Pb})$ reactions at $4,6,8$, 11, $20 \mathrm{~A} \mathrm{GeV}$ (left part) and at 30, 40,80,160 A GeV and $\sqrt{s}=200 \mathrm{GeV}$ (right part) with the data (full and open symbols) from Refs. [10,36,37,42-44]. ${ }^{2}$ At the lowest energy of $4 A \mathrm{GeV}$ the agreement between the transport approaches and the data is still acceptable, however, severe deviations are visible in the $K^{ \pm}$spectra at top AGS, SPS, and RHIC energies. Note that the $\pi^{ \pm}$spectra are reasonably described at all energies, while the inverse slope $T$ of the $K^{ \pm}$transverse mass spectra is underestimated severely by about the same amount in both transport approaches (within statistics). The increase of the inverse $K^{ \pm}$slopes in heavy ion collisions with respect to $p p$ collisions, which is generated by rescatterings of produced hadrons in the transport models, is only small because the elastic meson-baryon scattering is strongly forward peaked (in HSD and UrQMD 2.0), and therefore gives little additional transverse momentum especially at midrapidity.

\section{TESTING ALTERNATIVE SCENARIOS}

In this section we will investigate different hadron/string scenarios, which might contribute to the increase of the inverse slope parameter of the $K^{ \pm}$mesons in central $\mathrm{Au}+\mathrm{Au}$ collisions and are not included in the "default" versions of the transport approaches.

\section{A. In-medium potentials}

The question comes up whether the discrepancies shown in Fig. 7 might be due to conventional hadronic medium effects. In fact, the $m_{T}$ slopes of kaons and antikaons at SIS energies $(1.5-2 \mathrm{~A} \mathrm{GeV})$ were found to differ significantly [54]. As argued in [24] the different slopes could be traced back to repulsive potentials of kaons with nucleons, which lead to a hardening of the $K^{+}$spectra, and attractive antikaonnucleon potentials, which lead to a softening of the $K^{-}$spectra. However, the effect of such potentials was calculated within HSD and was found to be of minor importance at AGS and SPS energies [24] since the meson densities are comparable to or even larger than the baryon densities at AGS energies and above.

Additional self-energy contributions stem from $K^{ \pm}$interactions with mesons, which become increasingly important for higher bombarding energies; however, $s$-wave kaon-pion interactions are weak due to chiral symmetry arguments, and $p$-wave interactions such as $\pi+K \leftrightarrow K^{*}$ transitions are sup-

\footnotetext{
${ }^{2}$ Note that all data from the NA49 Collaboration at $30 \mathrm{~A} \mathrm{GeV}$ have to be considered as "preliminary."
} 

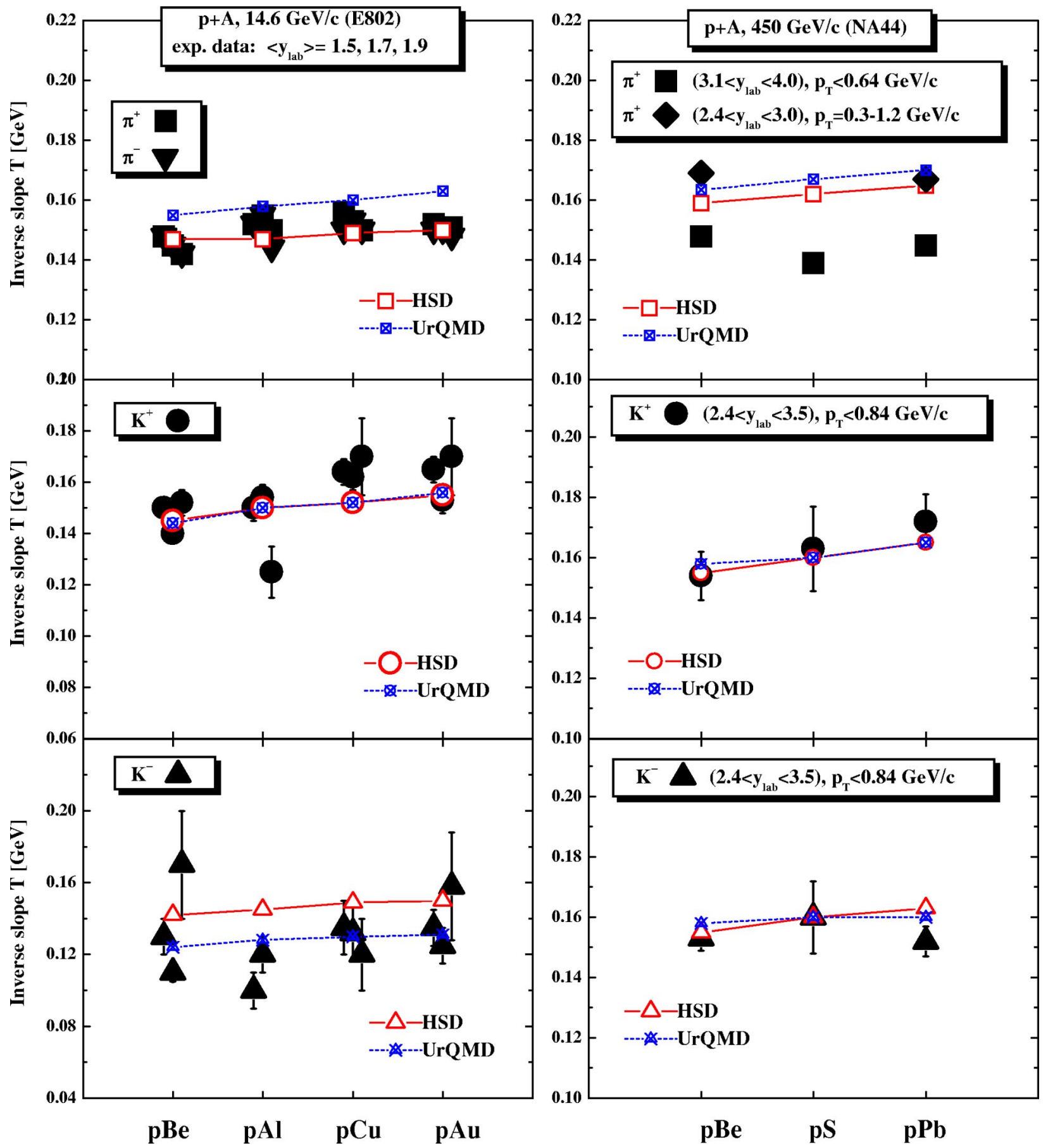

FIG. 6. (Color online) Inverse slope parameters $T$ for $\pi^{+}, K^{+}$, and $K^{-}$at midrapidity form $p A$ reactions at $14.6 \mathrm{GeV} / c(A=\mathrm{Be}, \mathrm{Al}, \mathrm{Cu}$, $\mathrm{Au}$ )—left part and at $450 \mathrm{GeV} / c(A=\mathrm{Be}, \mathrm{S}, \mathrm{Pb})$-right part, from HSD (open symbols connected by solid lines) and UrQMD 1.3(2.0) (open symbols with crosses connected by dashed lines $)$. The full symbols in the left part correspond to the midrapidity data $\left(\left\langle y_{\text {lab }}\right\rangle=1.5,1.7,1.9\right)$ form the E802 Collaboration [52], in the right part to the NA44 data [53] at $2.4 \leqslant y_{\mathrm{lab}} \leqslant 3.5, p_{T} \leqslant 0.84 \mathrm{GeV} / c$ for $K^{+}, K^{-}$and at $2.4 \leqslant y_{\mathrm{lab}}$ $\leqslant 3.0, p_{T}=0.3-1.2 \mathrm{GeV} / c$ (full diamonds) and $3.1 \leqslant y_{\text {lab }} \leqslant 4.0, p_{T} \leqslant 0.64 \mathrm{GeV} / c$ (full squares) for $\pi^{+}$.

pressed substantially by the approximately "thermal" pion spectrum. A recent study on the kaon potentials in hot pion matter gives kaon mass shifts of about $-52 \mathrm{MeV}$ and vector potentials of $\sim+49 \mathrm{MeV}$ [55] for a pion gas at temperature $T=170 \mathrm{MeV}$. We have employed even slightly larger $K^{ \pm}$potentials in dynamical HSD calculations and achieved a hardening of the $K^{ \pm}$spectra by less than $10 \%$.

\section{B. Overlapping strings}

Furthermore, we have pursued the idea of Refs. [33,56] that the $K^{ \pm}$spectra could be hardened by string-string interactions, which increase the effective string tension $\sigma$ and thus the probability to produce mesons at high $m_{T}[30,56]$. A related suggestion has been put forward in [57]. 

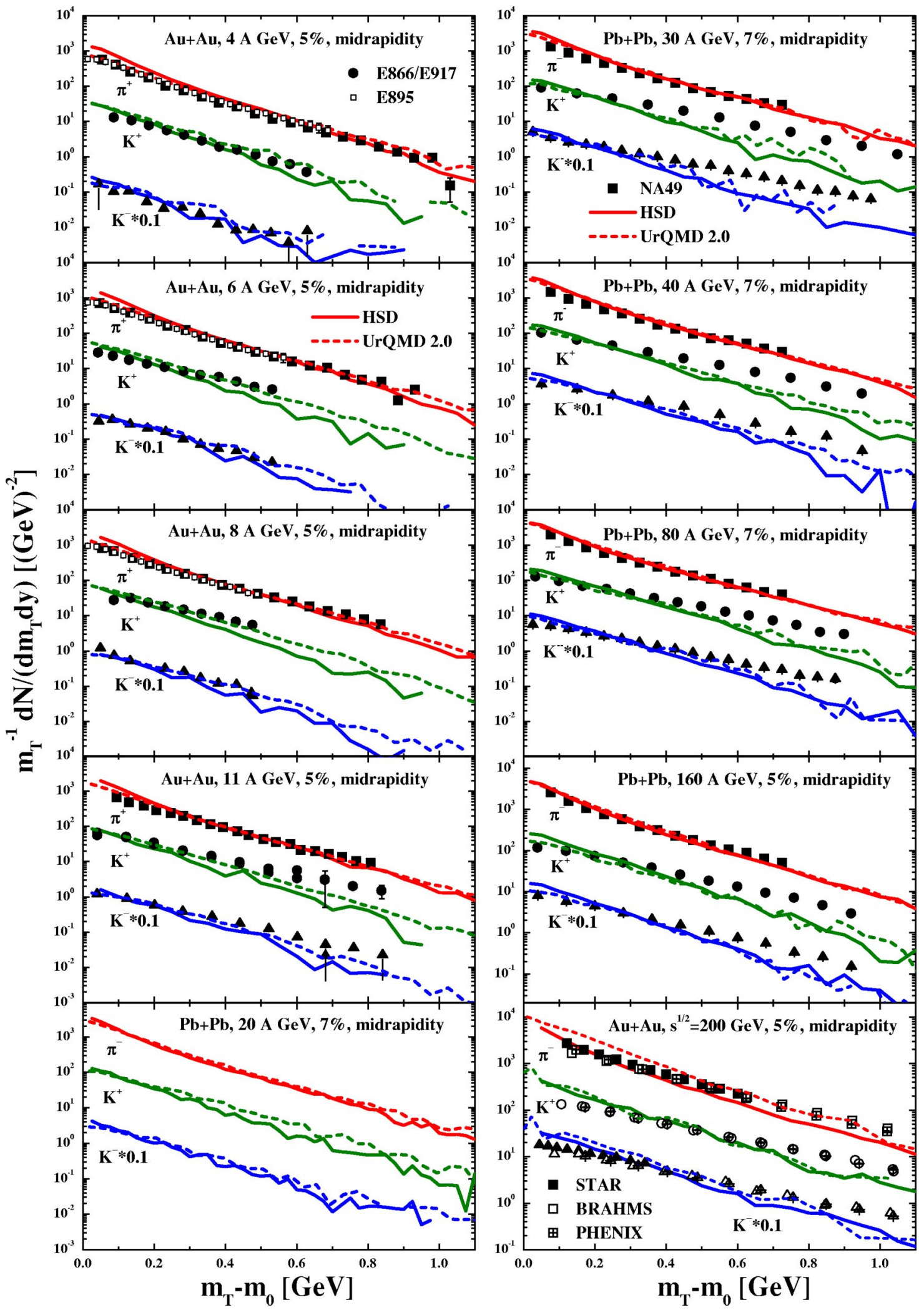

FIG. 7. (Color online) Comparison of transverse mass spectra at midrapidity from HSD (solid lines) and UrQMD 2.0 (dashed lines) for $\pi^{ \pm}, K^{+}$, and $K^{-}(\times 0.1)$ from central $\mathrm{Au}+\mathrm{Au}(\mathrm{Pb}+\mathrm{Pb})$ reactions at $4,6,8,11,20 \mathrm{~A} \mathrm{GeV}$ (left part) and at $30,40,80,160 \mathrm{~A} \mathrm{GeV}$ and $\sqrt{s}$ $=200 \mathrm{GeV}$ (right part) with the data (full and open symbols) from Refs. [10,36,37,42-44]. 
In order to estimate the largest possible effect of stringstring interactions we have assumed that for two overlapping strings, the string tension $\sigma$ is increased by a factor of 2 , for three overlapping strings by a factor of 3 , etc. Here the overlap of strings is defined by geometrically assuming a transverse string radius $R_{s}$, which according to the studies in Ref. [58] should be $R_{s} \leqslant 0.25 \mathrm{fm}$. Additionally, an overlap of the strings in longitudinal direction is required which, however, does not involve any new parameter since the dynamics of the string ends is directly available from the HSD transport calculations. Based on these assumptions (and $R_{s}=0.25 \mathrm{fm}$ as in Ref. [57]), we find only a small increase of the inverse slope parameters at AGS energies, where the string densities are low [dashed lines in Fig. 8 in comparison to the default calculations (solid lines)]. At SPS and RHIC energies the model gives a hardening of the spectra by about $15 \%$ which, however, is still too low in comparison to the data in Fig. 7. Despite of the large number of initial strings in central $\mathrm{Au}$ + Au collisions at SPS or RHIC energies, their average eigenvolume (in the rest frame at breakup) is low $\left(\approx 0.3 \mathrm{fm}^{3}\right)$ compared to the eigenvolume of a nucleon $\left(\approx 2 \mathrm{fm}^{3}\right)$ due to the fast string fragmentation process. Consequently the critical density of color neutral strings may be about 7 times higher than the critical density of nucleons with respect to a phase transition induced by percolation [59].

\section{Parton elastic scattering}

We have examined another possibility, which we denote as "parton elastic scattering." To this end we have included elastic scattering of unformed hadrons in the initial phase of the reaction-before they actually evolve to their full hadronic size-employing the elastic pion-nucleon cross section per quark degree of freedom. The results of these simulations are shown in Fig. 8 by the dotted lines, which give a much better reproduction of the experimental spectra at $\sqrt{s}$ $=200 \mathrm{GeV}$ that are even slightly overestimated. The drawback on the other side is that the hadron rapidity spectra now are overestimated by $\sim 20 \%$ relative to the default calculation (and the experimental data). The increase in the slope parameter especially for $K^{ \pm}$spectra at AGS and SPS energies, however, is only moderate and a significant discrepancy remains with respect to the experimental spectra.

\section{Isotropic decay of strings from meson-baryon collisions}

Following a further idea of Ref. [60] we explore the possibility that the fragmentation of strings from $m B$ collisions modifies in the dense and hot medium. An extreme modification amounts to assume an isotropic decay of $m B$ strings - as employed in the RQMD model [33]—which leads to a reduction of longitudinal hadron momenta and an enhancement of their transverse momenta. The results of these simulations are displayed in Fig. 8 for the transverse mass spectra of pions, kaons, and antikaons (dotted-dashed lines). Apparently, this modified fragmentation function leads to much better results in comparison to the experiment (cf. dotted-dashed lines in Fig. 8).

The drawback to this scenario, however, is that the approximate agreement with the longitudinal rapidity distribu- tions for $\pi^{-}, K^{+}, K^{-}$, and protons is destroyed as demonstrated in Fig. 9 for $5 \%$ central $\mathrm{Pb}+\mathrm{Pb}$ reactions at $160 \mathrm{~A} \mathrm{GeV}$. Here the solid lines correspond to the default HSD calculations, whereas the dashed lines stand for the calculations with an isotropic decay of $m B$ strings. Obviously, the latter scenario leads to an enhanced stopping of the nuclei and larger meson production, which is not supported very much by the data. We admit that a slightly more isotropic decay of the $m B$ strings (with respect to the default calculations) would be favored by the experimental data; however, we do not aim at fitting data here. We mentionwithout explicit representation-that the comparison shown in Fig. 9 for central collisions at $160 \mathrm{~A} \mathrm{GeV}$ holds qualitatively also at AGS and RHIC energies.

\section{E. High mass baryon resonances (UrQMD 2.1)}

A further possibility to harden the meson and nucleon spectra in a coupled-channel transport approach is to introduce higher mass baryon resonances with masses $>2 \mathrm{GeV}$ as in RQMD [33], that decay isotropically and dominantly couple to the $\pi N$ channel and not to multipion+ nucleon channels. As described in Sec. II, the version UrQMD 2.1 has been set up to incorporate this possibility and to explore its consequences. Since the "massive" resonances decay isotropically in their rest frame, this leads to enhanced transverse flow as found in the previous subsection. The penalty of enhanced hadron production (cf. Fig. 9) is cured in UrQMD 2.1, since the high mass resonance decays produce $25 \%$ less particles as compared to a string of the same invariant mass. In this way one can produce larger hadron transverse momenta in the transport calculations without increasing the meson multiplicities on average. Such a prescription fits the data in the AGS and SPS energy regime very well (similar to RQMD [33,62]). However, the open question remains: can such states be identified in mesonbaryon scattering and, furthermore, would they survive in a dense hadronic medium?

In Fig. 10 we show the transverse mass spectra at midrapidity for $\pi^{-}, K^{+}$, and $K^{-}(\times 0.1)$ from $5 \%$ central $\mathrm{Au}$ $+\mathrm{Au}(\mathrm{Pb}+\mathrm{Pb})$ reactions at $11,160 \mathrm{~A} \mathrm{GeV}$ and $\sqrt{s}=200 \mathrm{GeV}$ calculated for different scenarios in UrQMD: the dashed lines correspond to the default calculations (UrQMD 2.0) and the solid lines to simulations with high mass baryon resonances (UrQMD 2.1). Indeed, the description of the pion and $K^{ \pm}$improves substantially in UrQMD 2.1 relative to UrQMD 2.0 (and HSD) at AGS and SPS energies. However, the penalty here is that now the spectra at the top RHIC energy are overestimated at high transverse mass for pions and antikaons.

Moreover, the improvement in the spectral slope for pions and kaons at AGS and SPS energies in UrQMD 2.1 does not go along with an improvement in the rapidity spectra as shown in Fig. 11. Though the $\pi^{-}$rapidity distribution can be well described for $5 \%$ central $\mathrm{Pb}+\mathrm{Pb}$ reactions at $160 \mathrm{~A} \mathrm{GeV}$ due to the reduction of pion production in the decay scheme of the high mass states, the rapidity spectra of $K^{ \pm}$mesons are also lower in UrQMD 2.1 (solid lines) compared to UrQMD 2.0 (dashed lines) and the data. The proton stopping at 


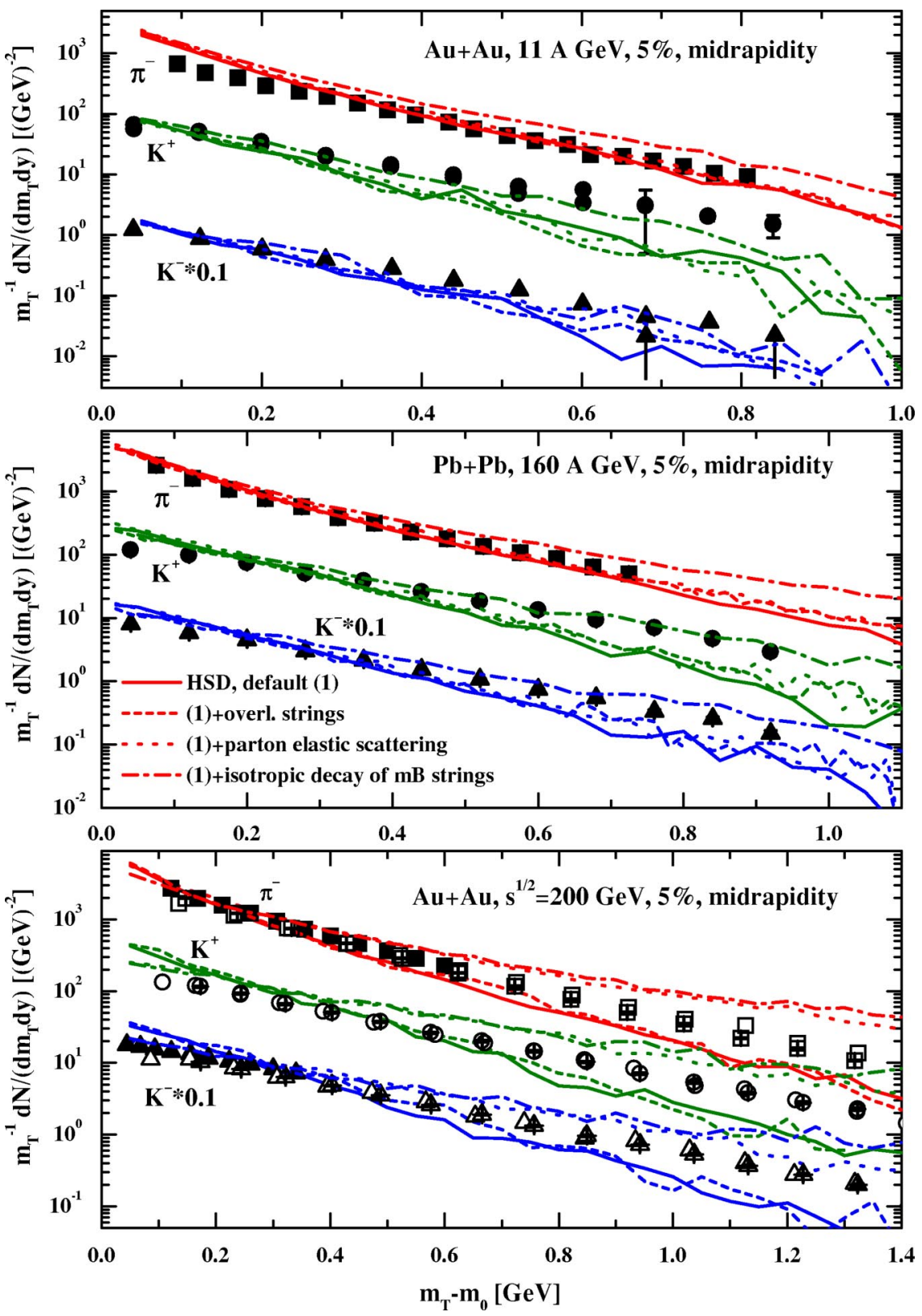

FIG. 8. (Color online) The transverse mass spectra at midrapidity for $\pi^{-}, K^{+}$, and $K^{-}(\times 0.1)$ from $5 \%$ central $\mathrm{Au}+\mathrm{Au}(\mathrm{Pb}+\mathrm{Pb})$ reactions at $11,160 \mathrm{~A} \mathrm{GeV}$, and $\sqrt{s}=200 \mathrm{GeV}$ calculated for different scenarios in HSD: The solid lines correspond to the default calculations, the dashed lines to simulations with overlapping strings, the dotted lines reflect calculations including "parton elastic scattering" and the dotted-dashed lines arise from calculations with an isotropic decay of meson-baryon $(m B)$ strings (see text). The experimental data are the same as in Fig. 7.

midrapidity is not much affected by the introduction of the high mass states since they do not couple to the baryonbaryon channel.

We conclude that the recipe of high mass states-as incorporated in UrQMD similar to RQMD_-does not solve the problem with the $K^{+} / \pi^{+}$ratio.

\section{CRONIN ENHANCEMENT IN $p A$ AND $A A$ COLLISIONS}

The Cronin effect dates back to measurements of transverse hadron spectra from $p A$ collisions at the SPS and ISR
$[63,64]$ and relates to a (moderate) enhancement of transverse momentum spectra with increasing target mass $A$. Though the microscopic origin of this effect is not fully understood, most current interpretations are based on semihard initial state gluon radiation of the propagating quarks in the medium prior to a hard interaction vertex (cf., e.g., [65]). In this way, the initial transverse momentum distribution of partons is extended to a higher average $\left\langle k_{T}^{2}\right\rangle$. We discard a further discussion of its physical origin and proceed with effective simulations following Ref. [25]. In the latter work it was suggested that such an enhancement of the intrinsic quark transverse momentum spread $\left\langle k_{T}^{2}\right\rangle$ might be simulated in the 

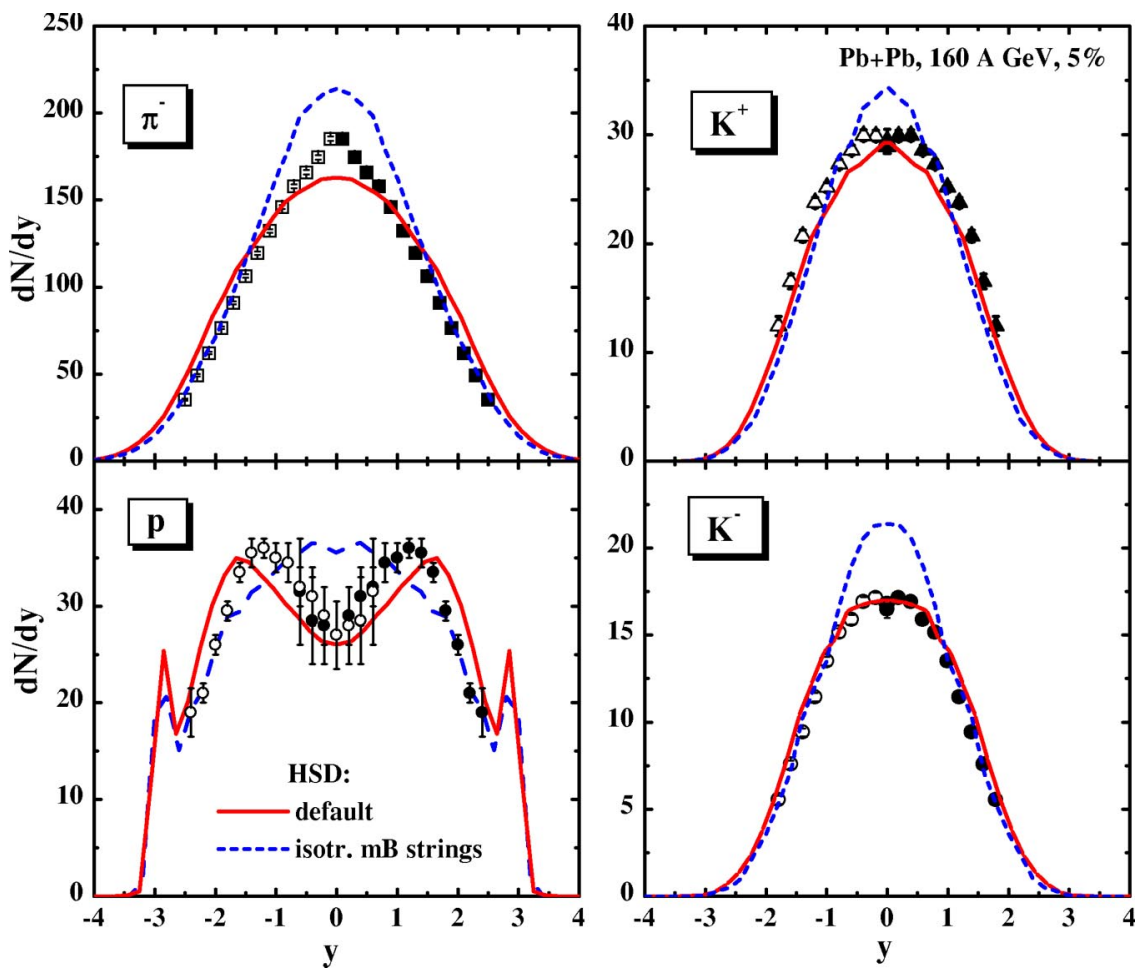

FIG. 9. (Color online) The rapidity distribution for $\pi^{-}, K^{+}, K^{-}$, and protons from $5 \%$ central $\mathrm{Pb}+\mathrm{Pb}$ reactions at $160 A \mathrm{GeV}$. The solid lines correspond to the default HSD calculations, whereas the dashed lines show the calculations with an isotropic decay of strings from $m B$ collisions (see text). The experimental data are taken from Refs. [9,61].

transport approach by increasing the average transverse momentum spread of the quarks $\left\langle k_{T}^{2}\right\rangle$ with the number of previous collisions of primary nucleons $N_{\text {prev }}$ as

$$
\left\langle k_{T}^{2}\right\rangle=\left\langle k_{T}^{2}\right\rangle_{p p}\left(1+\alpha N_{\text {prev }}\right) \text {. }
$$

The parameter $\alpha \approx 0.4$ has been determined in Ref. [25] by comparing to the experimental data for $d+\mathrm{Au}$ collisions $[12,13]$ at $\sqrt{s}=200 \mathrm{GeV}$. This assumption is in line with an independent analysis within the Glauber model by Papp et al. [66]. Note, that $N_{\text {prev }}$ is not a further parameter of the model, but calculated dynamically in the transport approach.

Whereas the different alternative scenarios discussed in Sec. V relate to various model assumptions, which are not directly controlled by experimental data, the initial state Cronin enhancement is an established experimental fact. Consequently, it should be incorporated in future transport approaches (as in Ref. [25]). For further details we refer the reader to the latter publication.

\section{$A+A$ reactions with initial state Cronin enhancement}

Our results for the transverse mass spectra at midrapidity for $\pi^{-}, K^{+}$, and $K^{-}(\times 0.1)$ from $5 \%$ central $\mathrm{Au}+\mathrm{Au}(\mathrm{Pb}$ $+\mathrm{Pb})$ reactions at $11 A \mathrm{GeV}, 160 A \mathrm{GeV}$ and $\sqrt{s}=200 \mathrm{GeV}$ as calculated within HSD including the Cronin effect (2) (solid lines) are presented in Fig. 12 in comparison to the default calculations (dashed lines). The experimental data are the same as in Fig. 7. The description of the spectra becomes rather good at RHIC energies, improves substantially at the SPS energy of $160 \mathrm{~A} \mathrm{GeV}$, but does not give any significant change at $11 A \mathrm{GeV}$. Consequently, the "prehadronic" Cronin effect-as implemented via Eq. (2) - is not responsible for the dramatic increase of the $K^{ \pm}$slopes at AGS energies. Our calculations, however, show that a hardening of the transverse mass spectra should not only be attributed to the presence of collective flow, but also to the initial state semihard scattering of fast quarks in a dense medium; the latter effect becomes more important with increasing $\sqrt{s}$. A more detailed and quantitative analysis on the competition of initial state Cronin enhancement and collective flow from pressure gradients at SPS and RHIC energies we postpone to an upcoming study.

\section{INVERSE SLOPE PARAMETERS AND $\left\langle p_{T}\right\rangle$}

Our findings are summarized in Fig. 13, where the dependence of the inverse slope parameter $T$ [defined by Eq. (1)] on $\sqrt{s}$ is shown and compared to experimental data (partly preliminary) from Refs. [10,36,41-44,67] for central $\mathrm{Au}$ $+\mathrm{Au}(\mathrm{Pb}+\mathrm{Pb})$ collisions (1.h.s.) and $[44,68,69]$ for $p p$ collisions (r.h.s.). The upper and lower solid lines (with open circles) on the 1.h.s. of Fig. 13 correspond to results from HSD calculations, where the upper and lower limits are due to fitting the slope $T$ itself, an uncertainty in the repulsive $K^{ \pm}$-pion potential or the possible effect of string overlaps. The solid lines with stars correspond to the HSD calculations with the Cronin effect (2). The dashed lines with open upper triangles represent slope parameters from UrQMD 1.3, the dotted-dashed lines with open lower triangles correspond to UrQMD 2.0 results, which are well within the limits ob- 

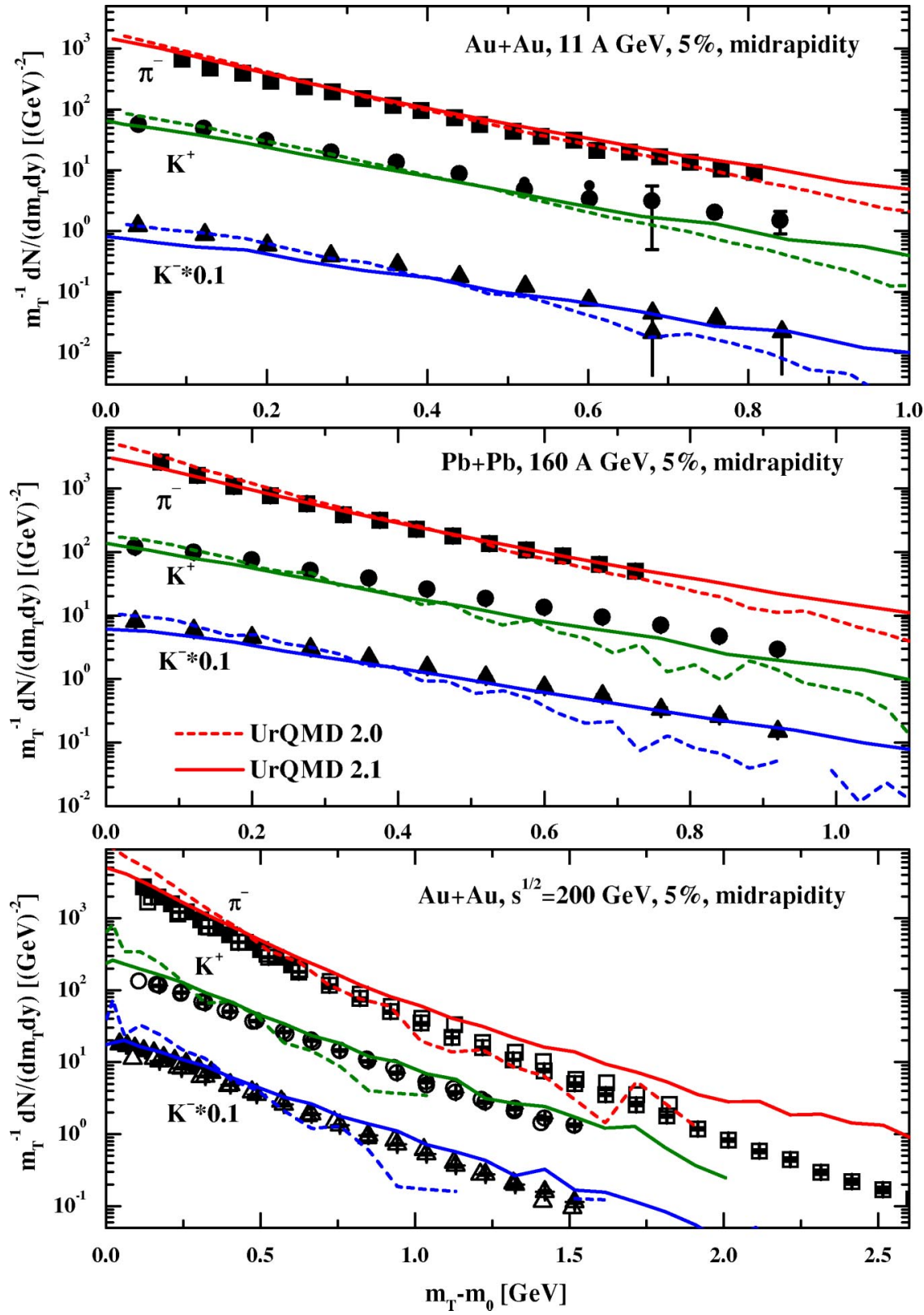

FIG. 10. (Color online) The transverse mass spectra at midrapidity for $\pi^{-}, K^{+}$, and $K^{-}(\times 0.1)$ from $5 \%$ central $\mathrm{Au}+\mathrm{Au}(\mathrm{Pb}$ $+\mathrm{Pb})$ reactions at $11,160 \mathrm{~A} \mathrm{GeV}$, and $\sqrt{s}=200 \mathrm{GeV}$ calculated for different scenarios in UrQMD: the dashed lines correspond to the default calculations (UrQMD 2.0), the solid lines to simulations with high mass baryon resonances (UrQMD 2.1). The experimental data are the same as in Fig. 7. tained from the different HSD calculations without the Cronin enhancement. The dotted lines with crosses show the UrQMD 2.1 results that incorporate the high mass resonance states.

The slope parameters from $p p$ collisions (r.h.s. in Fig. 13) are seen to increase smoothly with energy both in the experiment (full symbols) and in the HSD calculations (full lines with open circles). The error bars related to our calculations are due to an uncertainty in extracting the slope parameter from different $m_{T}$ intervals. The UrQMD 1.3 results are shown as open upper triangles connected by the solid line and are systematically lower than the slopes from HSD at all energies. When including jet production and fragmentation via PYTHIA in UrQMD 2.0 (dotted-dashed lines with open lower triangles) the results become similar to HSD above $\sqrt{s}=10 \mathrm{GeV}$ demonstrating the importance of jets in $p p$ reactions at high energy.
Coming back to the slope parameters of $K^{ \pm}$mesons for central $\mathrm{Au}+\mathrm{Au} / \mathrm{Pb}+\mathrm{Pb}$ collisions (1.h.s. of Fig. 13) we find that the Cronin initial state enhancement indeed improves the description of the data at RHIC energies, however, it does not give any sizeable enhancement at AGS energies. Here UrQMD 2.1 (dashed lines with crosses) with the high mass resonance states performs better for $K^{+}$mesons, but overestimates the $K^{-}$slopes at AGS as well as upper SPS energies.

An alternative way of representing the data is provided by the excitation function for the average transverse momentum $\left\langle p_{T}\right\rangle$ for pions (1.h.s.) and kaons (r.h.s.) in Fig. 14 for the different transport models in comparison to the data available. HSD: upper and lower solid lines with open circles, UrQMD 2.0: dotted-dashed lines with open lower triangles and UrQMD 2.1: dotted lines with crosses; the data are from Refs. [42-44,70,71]. The dotted line with the open diamonds (r.h.s.) shows $\left\langle p_{T}\right\rangle$ for $K^{-}$from UrQMD 2.1. Furthermore, the 

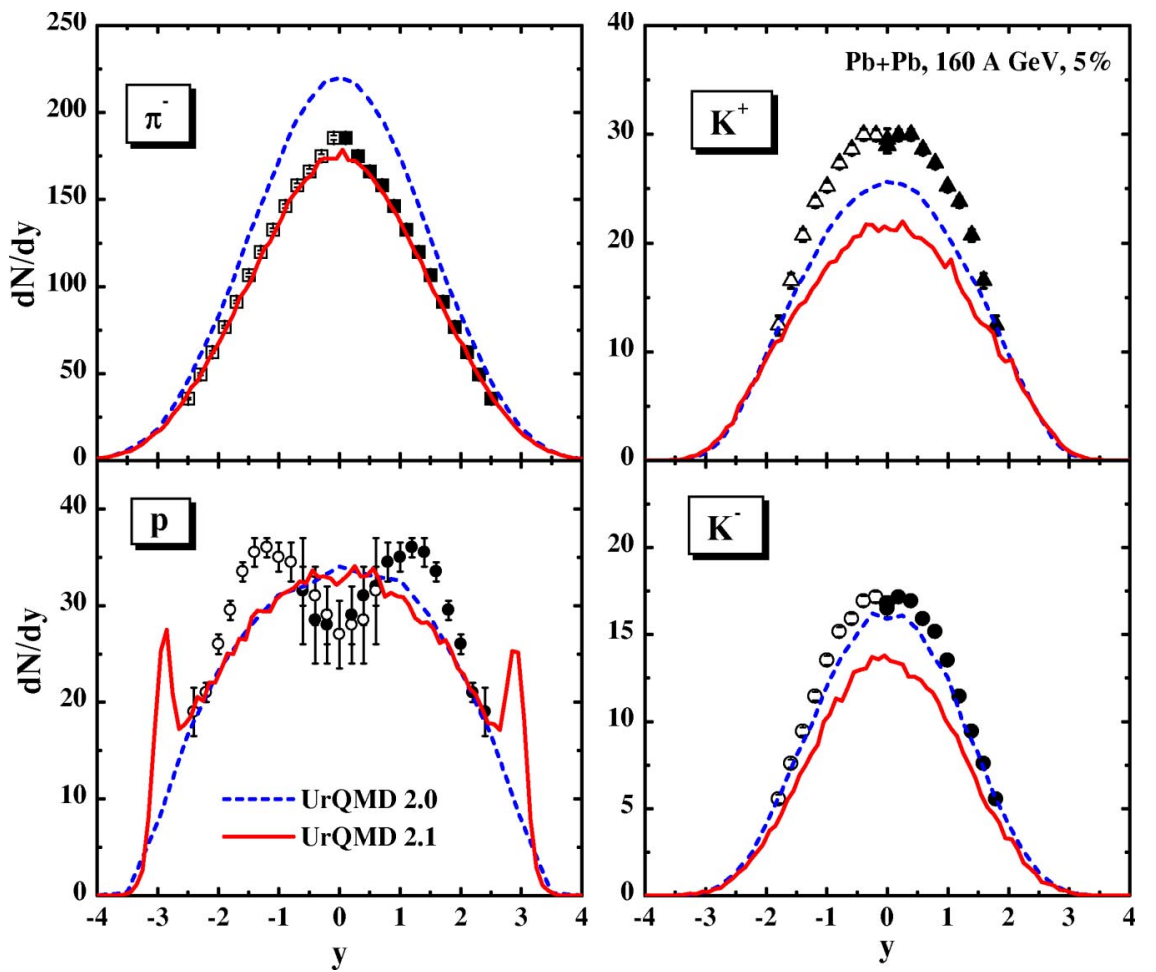

FIG. 11. (Color online) The rapidity distribution for $\pi^{-}, K^{+}, K^{-}$, and protons from $5 \%$ central $\mathrm{Pb}$ $+\mathrm{Pb}$ reactions at $160 \mathrm{~A} \mathrm{GeV}$. The dashed lines correspond to the default calculations from UrQMD 2.0, whereas the solid lines show the calculations with high mass baryon resonances (UrQMD 2.1). The experimental data are taken from Refs. [9,61].

solid lines with stars correspond to the HSD calculations with the Cronin effect, which gives a good description of the average $\left\langle p_{T}\right\rangle$ in case of pions and significantly improves the average $\left\langle p_{T}\right\rangle$ for kaons as a function of $\sqrt{s}$ (with respect to default HSD and UrQMD 2.0 calculations). The UrQMD 2.1 calculations overestimate $\left\langle p_{T}\right\rangle$ for pions at all energies by $10-15 \%$ as well as for $K^{-}$at AGS and SPS energies in line with the trends from Fig. 13; however, the average $\left\langle p_{T}\right\rangle$ for $K^{+}$mesons is rather well described.

\section{THERMODYNAMICS IN THE $T-\mu_{B}$ PLANE}

This still leaves us with the question of the origin of the rapid increase of the $K^{ \pm}$slopes with invariant energy for central $\mathrm{Au}+\mathrm{Au}$ collisions at AGS energies and the constant slope at SPS energies (the "step"), which is missed in all transport approaches presently employed. We recall that higher transverse particle momenta either arise from repulsive self-energies - in mean-field dynamics-or from collisions, which reduce longitudinal momenta in favor of transverse momenta [4,72]. As shown above in Fig. 13, conventional hadron self-energy effects and (pre-)hadronic and hadronic binary collisions are insufficient to describe the dramatic increase of the $K^{ \pm}$slopes as a function of $\sqrt{s}$ as well as of the initial state Cronin enhancement. Only when including high mass resonances in UrQMD 2.1-with a particular decay pattern-we may end up with reasonable results for $\mathrm{K}^{+}$mesons, however, fail more severely for pions as well as antikaons. These failures of $10-15 \%$ indicate an additional mechanism for the generation of the pressure that is observed experimentally, especially at AGS energies.

We speculate-following the previous study [20]—-that partonic degrees of freedom should be responsible for this effect already at $\sim 5 A \mathrm{GeV}$. Our arguments here are based on a comparison of the thermodynamic parameters $T$ and $\mu_{B}$ extracted from the transport models in the central overlap regime of $\mathrm{Au}+\mathrm{Au}$ collisions [28] with the experimental systematics on chemical freeze-out configurations [73] in the $T, \mu_{B}$ plane. The solid line in Fig. 15 characterizes the universal chemical freeze-out line from Cleymans and Redlich [73], whereas the full dots with errorbars denote the experimental chemical freeze-out parameters-determined from the thermal model fits to the experimental ratios-taken from Ref. [73]. The various symbols (in vertical sequence) represent temperatures $T$ and chemical potentials $\mu_{B}$ extracted from UrQMD 1.3 transport calculations in central $\mathrm{Au}$ $+\mathrm{Au}(\mathrm{Pb}+\mathrm{Pb})$ collisions at $21.3 A \mathrm{TeV}, 160,40$, and $11 A \mathrm{GeV}$ [28] as a function of the reaction time in the center of mass (from top to bottom). The open symbols denote nonequilibrium configurations and correspond to $T$ parameters extracted from the transverse momentum distributions, whereas the full symbols denote configurations in approximate pressure equilibrium in longitudinal and transverse direction.

During the nonequilibrium phase (open symbols) the transport calculations show much higher temperatures (or energy densities) than the experimental chemical freeze-out configurations at all bombarding energies $(\geqslant 11 A \mathrm{GeV})$. These numbers are also higher than the tricritical endpoints extracted from lattice QCD calculations by Karsch [74] (large open circle) and Fodor and Katz [7] (star with horizontal error bar). Though the QCD lattice calculations differ substantially in the value of $\mu_{B}$ for the critical endpoint, the critical temperature $T_{c}$ is closer to $160 \mathrm{MeV}$ in both calculations, while the energy density is of the order of $1 \mathrm{GeV} / \mathrm{fm}^{3}$ or even below. Nevertheless, this diagram shows that at RHIC energies one encounters more likely a cross over be- 

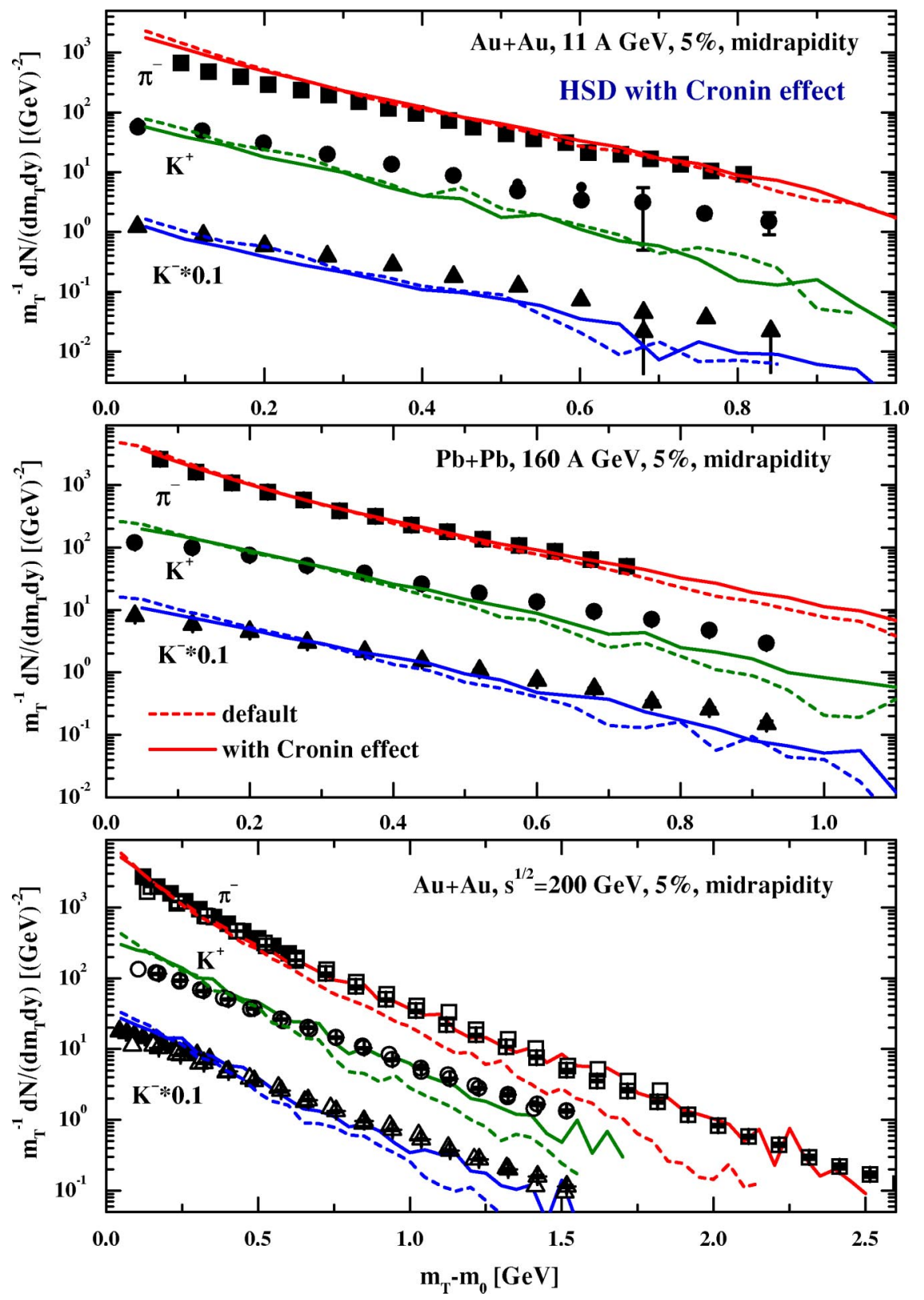

FIG. 12. (Color online) The transverse mass spectra at midrapidity for $\pi^{-}, K^{+}$, and $K^{-}(\times 0.1)$ from $5 \%$ central $\mathrm{Au}+\mathrm{Au}(\mathrm{Pb}$ $+\mathrm{Pb}$ ) reactions at $11,160 A \mathrm{GeV}$, and $\sqrt{s}=200 \mathrm{GeV}$ calculated within HSD including the Cronin effect (2) (solid lines) in comparison to the default calculations (dashed lines). The experimental data are the same as in Fig. 7.

tween the different phases when stepping down in temperature during the expansion phase of the hot fireball.

This situation might change at lower SPS or AGS (as well as at new GSI SIS-300 [75]) energies, where for sufficiently large chemical potentials $\mu_{B}$, the cross over should change to a first order transition [76], i.e., beyond the tricritical point in the $\left(T, \mu_{B}\right)$ plane. Nevertheless, Fig. 15 demonstrates that the transport calculation shows temperatures (energy densities) well above the phase boundary (horizontal line with errorbars from Ref. [7]) in the very early phase of the collisions, where hadronic interactions yield only a pressure $p$ $\sim 0.1-0.14 \epsilon$ (with $\epsilon$ denoting the energy density). We recall that for massless partonic degrees of freedom we have $p$ $\sim \epsilon / 3$ and consequently a much higher pressure. This argument is well in line with the studies on elliptic flow at RHIC energies, that is underestimated by $\sim 30 \%$ at midrapidity in the HSD approach [48] and by a factor of $\sim 2$ in the UrQMD
1.3 model [77]. Only strong prehadronic/partonic interactions might cure this problem.

As shown in Ref. [78], in order to describe the elliptic flow seen experimentally at RHIC in a parton cascade model, one has to employ parton cross sections up to $45 \mathrm{mb}$. However, such strong interactions are incompatible with perturbative QCD, which gives cross sections that are lower by more than one order of magnitude [78]. We speculate that such strong nonperturbative interactions on the partonic level are responsible for the large pressure generation in the very early phase of intermediate energy nucleus-nucleus collisions.

\section{SUMMARY}

Summarizing this work, we point out that baryon stopping and hadron production in central $\mathrm{Au}+\mathrm{Au}($ or $\mathrm{Pb}+\mathrm{Pb})$ colli- 


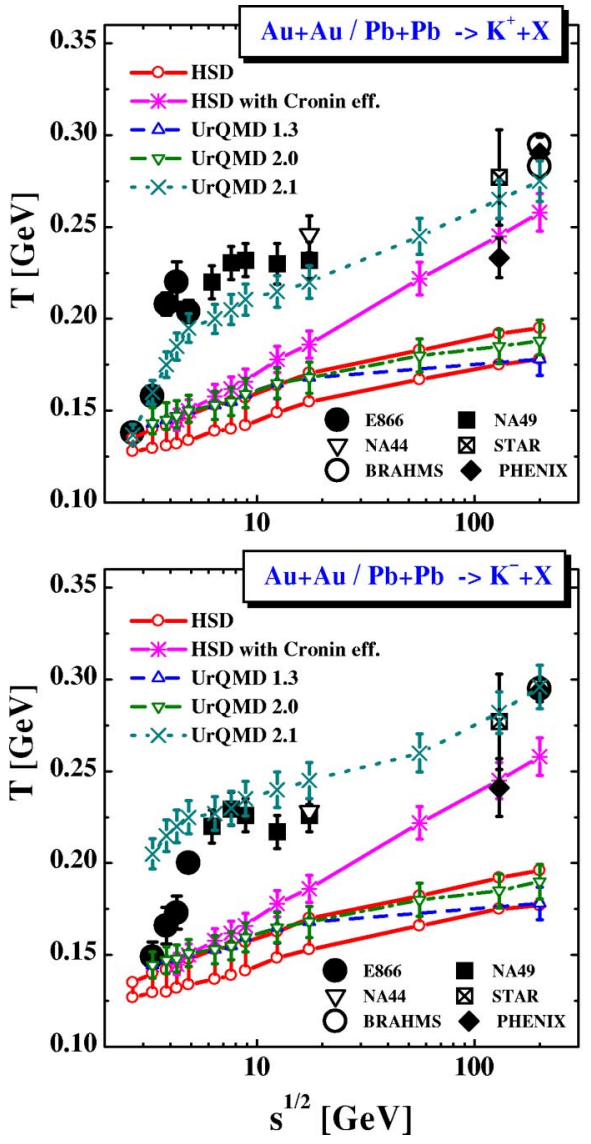

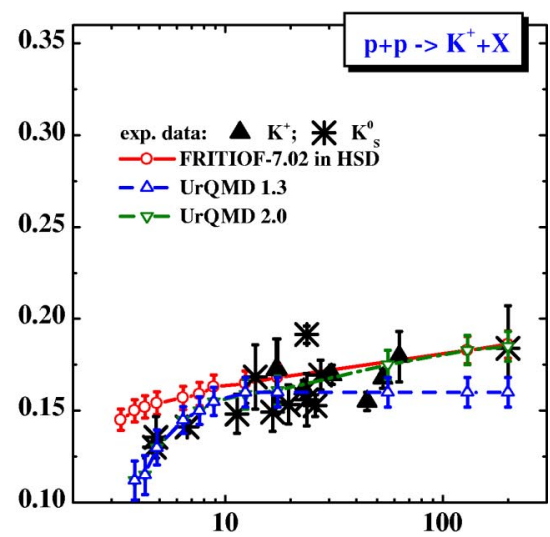

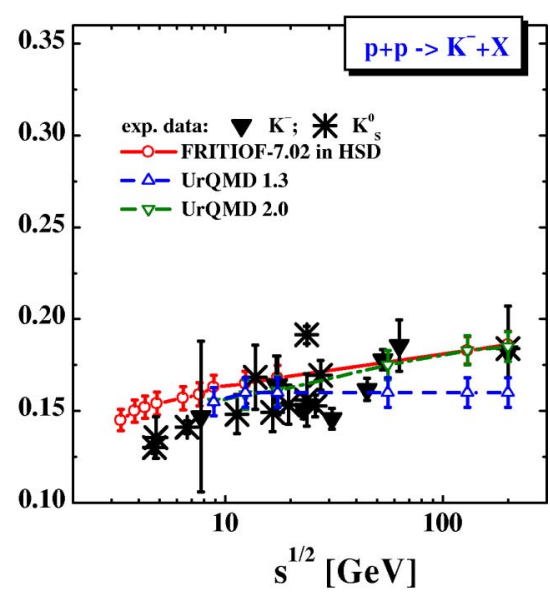

FIG. 13. (Color online) Comparison of the inverse slope parameters $T$ for $K^{+}$and $K^{-}$mesons from central $\mathrm{Au}+\mathrm{Au}(\mathrm{Pb}+\mathrm{Pb})$ collisions (1.h.s.) and $p p$ reactions (r.h.s.) at midrapidity as a function of the invariant enegry $\sqrt{s}$ from HSD (upper and lower solid line with open circles), UrQMD 1.3 (dashed lines with open upper triangles), UrQMD 2.0 (dot-dashed lines with open lower triangles), UrQMD 2.1 (dotted lines with crosses) with the data from Refs. [10,36,41-44,67] for $A A$ and $[44,68,69]$ for $p p$ collisions. The upper and lower solid lines in the left diagrams result from different limits of the HSD calculations as discussed in the text while the solid lines with stars correspond to HSD calculations with the Cronin initial state enhancement (2). sions is quite well described in the independent transport approaches HSD and UrQMD 2.0. Also the longitudinal rapidity distributions of protons, pions, kaons, antikaons, and hyperons are similar in both models and in reasonable agreement with available data. The exception are the pion rapidity spectra at the highest AGS energy and lower SPS energies, which are overestimated by both models [19]. As a consequence, the HSD and UrQMD 2.0 transport approaches un- derestimate the experimental maximum of the $K^{+} / \pi^{+}$ratio at 20-30 A GeV and UrQMD underestimates also the data at RHIC energies. This also holds for the new version UrQMD 2.1 introduced here, which incorporates high mass resonance states above $2 \mathrm{GeV}$ with a particular decay pattern. This recipe cures the problem of the overestimated pion yield at AGS and SPS energies, but also reduces the $K^{ \pm}$yield and therefore has only little effect on the $K^{+} / \pi^{+}$ratio.
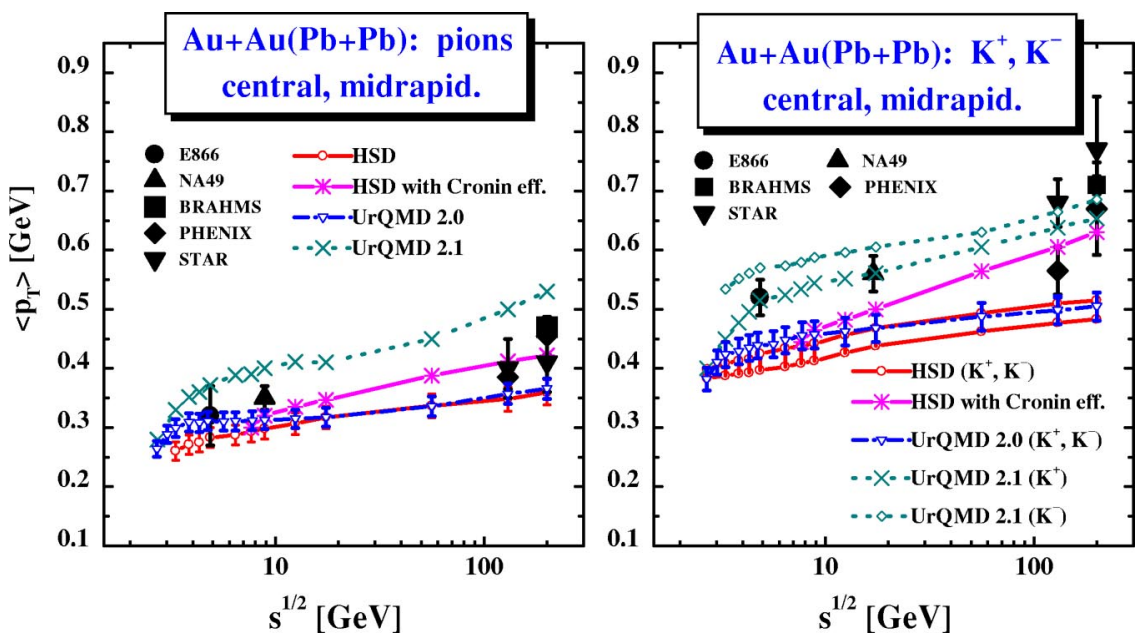

FIG. 14. (Color online) The excitation function for the average values of $p_{T}$ at midrapidity for pions (left) and kaons (right) calculated within HSD (upper and lower solid lines with open circles) and UrQMD 2.0 (dot-dashed lines with open lower triangles), UrQMD 2.1 (dotted lines with crosses) in comparison to the data from Refs. [42-44,70,71]. The solid lines with stars correspond to the HSD calculations with the Cronin effect (2). The dotted line with the open diamonds (right part) gives $\left\langle p_{T}\right\rangle$ for $K^{-}$from UrQMD 2.1. 


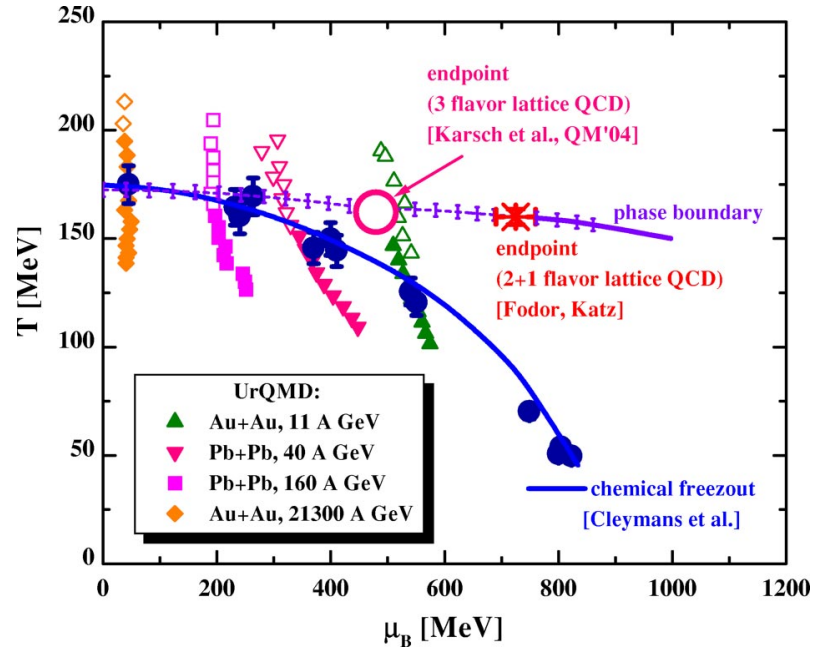

FIG. 15. (Color online) Schematic phase diagram in the $T-\mu_{B}$ plane. The solid line characterizes the universal chemical freeze-out line from Cleymans et al. [73] whereas the full dots (with errrorbars) denote the "experimental" chemical freeze-out parameters from Ref. [73]. The various symbols represent temperatures $T$ and chemical potentials $\mu_{B}$ extracted from UrQMD 1.3 transport calculations in central $\mathrm{Au}+\mathrm{Au}(\mathrm{Pb}+\mathrm{Pb})$ collisions at $21.3 A \mathrm{TeV}, 160$, 40, and $11 A \mathrm{GeV}$ [28] (see text). The large open circle and the star indicate the tricritical endpoints from lattice QCD calculations by Karsch et al. [74] and Fodor and Katz [7], respectively. The horizontal line with errorbars is the phase boundary from [7].

We have found that the inverse slope parameters $T$ for $K^{ \pm}$ mesons from the HSD and UrQMD 2.0 transport models are practically independent of system size from $p p$ up to central $\mathrm{Pb}+\mathrm{Pb}$ collisions and show only a slight increase with collision energy. The calculated transverse mass spectra are in reasonable agreement with the experimental results for $p p$ reactions at all bombarding energies investigated, as well as at central collisions of light nuclei $(\mathrm{C}+\mathrm{C}$ and $\mathrm{Si}+\mathrm{Si})$ (cf. Ref. [20]). The rapid increase of the inverse slope parameters of kaons for central collisions of heavy nuclei $(\mathrm{Au}+\mathrm{Au}$ or $\mathrm{Pb}+\mathrm{Pb}$ ) found experimentally in the AGS energy range, however, is not reproduced by both models in their default version (see Fig. 13).
We have, furthermore, discussed a couple of scenarios in Sec. V to improve the description of the experimental data. However, none of the alternative scenarios leads to a fully satisfactory reproduction of all the experimental data. It is worth pointing out that a substantial hardening of the $K^{ \pm}$ slope results from an initial state Cronin enhancement at higher SPS and RHIC energies, that should not be attributed to collective flow (or initial pressure). This Cronin enhancement-as known experimentally from $p+A$ reactions at SPS energies-does not signal a phase transition to a quark-gluon-plasma.

We thus have argued-in comparison to lattice QCD calculations at finite temperature and baryon chemical potential $\mu_{B}$ from Refs. $[7,74]$ as well as the experimental systematics in the chemical freeze-out parameters (cf. Fig. 15) - that the missing pressure should be generated in the early phase of the collision by nonperturbative partonic interactions because the strong hadronic interactions do not produce it in the later stages.

The interesting finding of our analysis is, that prehadronic/partonic degrees of freedom seem to play a substantial role in central $\mathrm{Au}+\mathrm{Au}$ collisions already at AGS energies above $\sim 5 A \mathrm{GeV}$. This finding is in line with the observation that the excitation function of the proton elliptic flow $v_{2}$ also indicates a softening of the equation of state with increasing energy at $4-5 \mathrm{~A} \mathrm{GeV}$ in $\mathrm{Au}+\mathrm{Au}$ collisions [79-81]. However, further detailed experimental and theoretical studies also on collective flow properties from nucleusnucleus collisions will be necessary to unravel the various aspects of relativistic many-body systems.

\section{ACKNOWLEDGMENT}

The authors acknowledge inspiring discussions with $\mathrm{K}$. Gallmeister, C. Greiner, C. M. Ko, Z. W. Lin, A. Peshier, K. Redlich, V. D. Toneev, and H. Weber. Furthermore, they are indepted to M. Gaździcki and J. Klay for providing experimental data in numerical form. E.L.B. is supported by Deutsche Forschungsgemeinschaft (DFG) and GSI. S.A.B. acknowledges support by RIKEN, Brookhaven National Laboratory, and DOE Grant Nos. DE-FG02-96ER40945 and DE-AC02-98CH10886 as well as an Outstanding Junior Investigator Award (DOE Grant No. DE-FG02-03ER41239). M.B. and M.R. are supported by GSI.
[1] Quark Matter 2002 [Nucl. Phys.A715, 1 (2003)].

[2] J. Rafelski and B. Müller, Phys. Rev. Lett. 48, 1066 (1982); P. Koch, B. Müller, and J. Rafelski, Phys. Rep. 142, 167 (1986).

[3] F. Karsch et al., Nucl. Phys. B502, 321 (2001).

[4] H. Stöcker and W. Greiner, Phys. Rep. 137, 277 (1986).

[5] W. Cassing et al., Nucl. Phys. A674, 249 (2000).

[6] H. Weber et al., Phys. Lett. B 442, 443 (1998).

[7] Z. Fodor and S. D. Katz, J. High Energy Phys. 0203, 014 (2002); Z. Fodor, S. D. Katz, and K. K. Szabo, Phys. Lett. B 568, 73 (2003).

[8] J. D. Bjorken, Phys. Rev. D 27, 140 (1983).

[9] S. V. Afanasiev et al., NA49 Collaboration, Phys. Rev. C 66,
054902 (2002).

[10] V. Friese et al., NA49 Collaboration, J. Phys. G 30, 119 (2004).

[11] J. L. Nagle and T. S. Ullrich, nucl-ex/0203007.

[12] S. S. Adler et al., PHENIX Collaboration, Phys. Rev. Lett. 91, 072303 (2003); Phys. Rev. C 69, 034910 (2004).

[13] J. Adams et al., STAR Collaboration, Phys. Rev. Lett. 91, 072304 (2003); 91, 172302 (2003).

[14] I. Arsene et al., Phys. Rev. Lett. 91, 072305 (2003).

[15] X. N. Wang, Phys. Rev. C 58, 2321 (1998).

[16] R. Baier et al., Annu. Rev. Nucl. Part. Sci. 50, 37 (2000).

[17] M. I. Gorenstein, M. Gaździcki, and K. Bugaev, Phys. Lett. B 
567, 175 (2003).

[18] M. Gazdzicki and M. I. Gorenstein, Acta Phys. Pol. B 30, 2705 (1999).

[19] H. Weber, E. L. Bratkovskaya, W. Cassing, and H. Stöcker, Phys. Rev. C 67, 014904 (2003).

[20] E. L. Bratkovskaya, S. Soff, H. Stöcker, M. van Leeuwen, and W. Cassing, Phys. Rev. Lett. 92, 032302 (2004).

[21] S. A. Bass et al., Prog. Part. Nucl. Phys. 42, 255 (1998).

[22] M. Bleicher et al., J. Phys. G 25, 1859 (1999).

[23] J. Geiss, W. Cassing, and C. Greiner, Nucl. Phys. A644, 107 (1998).

[24] W. Cassing and E. L. Bratkovskaya, Phys. Rep. 308, 65 (1999).

[25] W. Cassing, K. Gallmeister, and C. Greiner, Nucl. Phys A735, 277 (2004).

[26] The HSD transport code is available from http:// www.th.physik.uni-frankfurt.de/ brat/hsd.html

[27] P. Braun-Munzinger, J. Stachel, J. P. Wessels, and N. Xu, Phys. Lett. B 344, 43 (1995); 365, 1 (1996); P. Braun-Munzinger, J. Cleymans, H. Oeschler, and K. Redlich, Nucl. Phys. A697, 902 (2002)

[28] L. V. Bravina et al., Phys. Rev. C 60, 024904 (1999); Nucl. Phys. A698, 383 (2002).

[29] E. L. Bratkovskaya et al., Nucl. Phys. A675, 661 (2000).

[30] Z. W. Lin et al., Nucl. Phys. A698, 375 (2002).

[31] Particle Data Group, K. Hagiwara et al., Phys. Rev. D 66, 010001 (2002).

[32] H.-U. Bengtsson and T. Sjöstrand, Comput. Phys. Commun. 46, 43 (1987).

[33] H. Sorge, Phys. Rev. C 52, 3291 (1995).

[34] B. Andersson et al., Z. Phys. C 57, 485 (1993).

[35] W. Ehehalt and W. Cassing, Nucl. Phys. A602, 449 (1996).

[36] L. Ahle et al., E866 and E917 Collaboration, Phys. Lett. B 476, 1 (2000); 490, 53 (2000); Phys. Rev. C 58, 3523 (1998).

[37] J. L. Klay et al., E895 Collaboration, Phys. Rev. C 68, 054905 (2003).

[38] S. Ahmad et al., E891 Collaboration, Phys. Lett. B 382, 35 (1996); C. Pinkenburg et al., E866 Collaboration, Nucl. Phys. A698, 495c (2002).

[39] A. Mischke et al., NA49 Collaboration, J. Phys. G 28, 1761 (2002); Nucl. Phys. A715, 453 (2003).

[40] F. Antinori et al., WA97 Collaboration, Nucl. Phys. A661, 130c (1999).

[41] M. Gazdzicki et al., NA49 Collaboration, nucl-ex/0403023.

[42] D. Ouerdane et al., BRAHMS Collaboration, Nucl. Phys. A715, 478 (2003); J. H. Lee et al., J. Phys. G 30, S85 (2004).

[43] S. S. Adler et al., PHENIX Collaboration, Phys. Rev. C 69, 024904 (2004); 69, 034909 (2004).

[44] C. Adler et al., STAR Collaboration, nucl-ex/0206008; O. Barannikova et al., Nucl. Phys. A715, 458 (2003); K. Filimonov et al., hep-ex/0306056.

[45] A. Andronic and P. Braun-Munzinger, hep-ph/0402291.

[46] P. Crochet et al., FOPI Collaboration, J. Phys. G 27, 267 (2001)

[47] M. van Leeuwen et al., NA49 Collaboration, Nucl. Phys.
A715, 161 (2003).

[48] E. L. Bratkovskaya, W. Cassing, and H. Stöcker, Phys. Rev. C 67, 054905 (2003).

[49] H. G. Fischer et al., NA49 Collaboration, Nucl. Phys. A715, 118c (2003).

[50] V. Blobel et al., Nucl. Phys. B69, 454 (1974).

[51] V. Topor Pop, M. Gyulassy, J. Barrette, C. Gale, X. N. Wang, N. Xu, and K. Filimonov, Phys. Rev. C 68, 054902 (2003).

[52] T. Abbott et al., E802 Collaboration, Phys. Rev. D 45, 3906 (1992).

[53] H. Boggild et al., NA44 Collaboration, Phys. Rev. C 59, 328 (1999).

[54] A. Förster et al., KaoS Collab., J. Phys. G 28, 2011 (2002).

[55] B. V. Martemyanov et al., nucl-th/0212064.

[56] S. Soff et al., Phys. Lett. B 471, 89 (1999).

[57] J. Dias de Deus, E. G. Ferreiro, C. Pajares, and R. Ugoccioni, Phys. Lett. B 581, 156 (2004); hep-ph/0304068.

[58] J. Geiss et al., Phys. Lett. B 447, 31 (1999).

[59] E. G. Ferreiro, C. Pajares, and D. Sousa, Phys. Lett. B 422, 314 (1998); M. A. Braun and C. Pajares, Eur. Phys. J. C 16, 349 (2000).

[60] M. Bleicher et al., Phys. Lett. B 447227 (1999).

[61] C. E. Copper et al., NA49 Collaboration, Nucl. Phys. A661, 362c (1999).

[62] H. van Hecke et al., Phys. Rev. Lett. 81, 5764 (1998).

[63] J. W. Cronin et al., Phys. Rev. D 11, 3105 (1975).

[64] D. Antreasyan et al., Phys. Rev. D 19, 764 (1979).

[65] B. Z. Kopeliovich, J. Nemchik, A. Schäfer, and A. V. Tarasov, Phys. Rev. Lett. 88, 232302 (2002).

[66] G. Papp, P. Levai, and G. Fai, nucl-th/0307062.

[67] I. G. Bearden et al., NA44 Collaboration, Phys. Rev. C 66, 044907 (2002).

[68] I. Kraus et al., NA49 Collaboration, J. Phys. G 30, 5583 (2004).

[69] M. Kliemant, B. Lungwitz, and M. Gaździcki, hep-ex/ 0308002.

[70] L. Ahle et al., E866 Collaboration, Nucl. Phys. A610, 139c (1996).

[71] J. Bächler et al., NA49 Collaboration, Nucl. Phys. A661, 45c (1999).

[72] W. Cassing and U. Mosel, Prog. Part. Nucl. Phys. 25, 235 (1990).

[73] J. Cleymans and K. Redlich, Phys. Rev. C 60, 054908 (1999).

[74] F. Karsch, talk given in Quark Matter 2004, Oakland, 2004; http://qm2004.lbl.gov

[75] "An International Accelerator Facility for Beams of Ions and Antiprotons," http://www.gsi.de/GSI-Future/cdr/

[76] E. V. Shuryak, Nucl. Phys. A661, 119c (1999).

[77] M. Bleicher and H. Stöcker, Phys. Lett. B 526, 309 (2002).

[78] D. Molnar and M. Gyulassy, Nucl. Phys. A698, 379 (2002).

[79] P. Danielewicz et al., Phys. Rev. Lett. 81, 2438 (1998).

[80] P. K. Sahu and W. Cassing, Nucl. Phys. A721, 357 (2002).

[81] E. G. Nikonov, A. A. Shanenko, and V. D. Toneev, Heavy Ion Phys. 8, 89 (1998). 